\title{
Article
}

\section{Random attractors for semilinear reaction-diffusion equation with distribution derivatives and multiplicative noise on $\mathbb{R}^{n}$}

\author{
Fadlallah Mustafa Mosa1 ${ }^{1}$, Abdelmajid Ali Dafallah ${ }^{2}$, Eshag Mohamed Ahmed ${ }^{3}$, Mohamed Y. A. Bakhet ${ }^{4,5}$ \\ and Qiaozhen $\mathrm{Ma}^{4, *}$ \\ 1 Department of Mathematics and physics, Faculty of Education, University of Kassala, Kassala, Sudan. \\ 2 Faculty of Petroleum and Hydrology Engineering, Peace University, Almugled, West Kordofan, Sudan. \\ 3 Faculty of Pure and Applied Sciences, International University of Africa, Khartoum, Sudan. \\ 4 College of Mathematics and Statistics, Northwest Normal University, Lanzhou, Gansu 730070, China. \\ 5 College of Education, Rumbek University of Science and Technology, Rumbek, South Sudan. \\ * Correspondence: maqzh@nwnu.edu.cn
}

Received: 1 January 2020; Accepted: 10 March 2020; Published: 12 April 2020.

\begin{abstract}
In this paper, we investigate the existence of random attractors for a semilinear reaction-diffusion equation with a nonlinearity having a polynomial growth of arbitrary order $p-1(p \geq 2)$, and with distribution derivatives and multiplicative noise defined on unbounded domains. The random attractors are obtained in $L^{2}\left(\mathbb{R}^{n}\right)$ and $L^{p}\left(\mathbb{R}^{n}\right)$ respectively. The semilinear reaction-diffusion equation is recast as a continuous random dynamical system and asymptotic compactness for this demonstrated by using uniform a priori estimates for far-field values of solutions as well as the cut-off technique.
\end{abstract}

Keywords: Semilinear reaction-diffusion equation, random dynamical system, distribution derivatives, measures of noncompactness.

MSC: 53B20, 53C20, 53C60.

\section{Introduction}

$\mathbf{T}$ In this article, we investigated the existence of the $\left(L^{2}\left(\mathbb{R}^{n}\right), L^{2}\left(\mathbb{R}^{n}\right)\right)$-random attractor and the $\left(L^{2}\left(\mathbb{R}^{n}\right), L^{p}\left(\mathbb{R}^{n}\right)\right.$ )-random attractor for the following semilinear reaction-diffusion equation with distribution derivatives and multiplicative noise on $\mathbb{R}^{n}$ :

$$
d u+(\lambda u-\Delta u) d t=\left(f(x)-g(u)+D_{j} f^{j}\right) d t+b u \circ d W(t) ; \text { in } \mathbb{R}^{+} \times \mathbb{R}^{n}
$$

with the initial value condition

$$
u(x, 0)=u_{0}(x) ; \quad x \in \mathbb{R}^{n},
$$

where $-\Delta$ is the Laplacian operator with respect to the variable $x \in \mathbb{R}^{n}, u=(x, t)$ is a real function of $x \in \mathbb{R}^{n}$ and $t>\tau, u_{\tau}(x) \in L^{2}\left(\mathbb{R}^{n}\right), \lambda$ and $b$ are non-negative constants, $D_{j}=\frac{\partial}{\partial x_{j}}$ is distribution derivatives, $f^{j}, f \in L^{2}\left(\mathbb{R}^{n}\right)(\mathrm{j}=1,2, \ldots, \mathrm{n})(n \geq 3), W(t)$ is a two-sided real-valued Wiener process on a probability space $(\Omega, \mathcal{F}, P)$, (where $\Omega=\{\omega \in C(\mathbb{R}, \mathbb{R}): \omega(0)=0\}, \mathcal{F}$ is the Borel $\sigma$-algebra induced by the compact-open topology of $\Omega$, and $P$ is the corresponding Wiener measure on $\mathcal{F}$ ), $\circ$ denotes the Stratonovich sense in the stochastic term. We identify $\omega(t)$ with $W(t)$, i.e., is a Wiener process defined on a standard probability space $(\Omega, \mathcal{F}, P)$, where $\Omega=\{\omega \in C(\mathbb{R}, \mathbb{R}): \omega(0)=0\}$, and $\mathcal{F}$ is the Borel $\sigma$-algebra induced by the compact-open topology of $\Omega$ and $P$ is the corresponding Wiener measure on $\mathcal{F}$. And $g$ is a Lipschitz function which satisfies the following conditions:

$$
\begin{gathered}
g(0)=0,\left(g\left(s_{1}\right)-g\left(s_{2}\right)\right)\left(s_{1}-s_{2}\right) \geq \alpha_{0}\left|s_{1}-s_{2}\right|^{2} \quad \forall s_{1}, s_{2} \in \mathbb{R}, \\
\beta_{2}|s|^{p}-\delta_{2}|s|^{2} \leq g(s) s \leq \beta_{1}|s|^{p}+\delta_{1}|s|^{2}, \quad 2<p<\infty
\end{gathered}
$$


where $\alpha_{0}, \beta_{1}, \beta_{2}, \beta_{3}, \delta_{1}$ and $\delta_{2}$ are non-negative constants and

$$
\delta_{2}<\lambda
$$

A random attractor of a random dynamical system is a measurable and compact invariant random set attracting all the orbits. The notion of a random attractor is very useful for many infinite-dimensional random dynamical systems (RDS), see [1,2].

Many authors have studied the existence of a random attractor for an RDS. For instance, Crauel and Flandoli in [1] introduced the notion of a random attractor and obtained a general theorem on the existence of a random attractor for the RDS. Their theorem has been successfully applied to the stochastic reaction-diffusion equations and the stochastic Navier-Stokes equations.

The existence of random attractors without distribution derivatives has been studied by several authors, see [2-10] and the reference therein. Notice that the partial differential equations (PDEs) studied in these literatures are all defined on the bounded domains. In the case of unbounded domains, the existence of random attractors without distribution derivatives was established for the stochastic reaction-diffusion equation with additive noise in [11], and with multiplicative noise in [12].

Recently, in case of distribution derivatives on unbounded domains, the existence of global attractors was established for the deterministic reaction-diffusion equation with distribution derivatives in $[13,14]$, and for the stochastic reaction-diffusion equation with distribution derivatives and additive noise in [15], and with multiplicative noise in [16].

Furthermore, there are no results on random attractors for stochastic reaction-diffusion equation with distribution derivatives and multiplicative noise on unbounded domain in $\left(L^{2}\left(\mathbb{R}^{n}\right), L^{p}\left(\mathbb{R}^{n}\right)\right)$. It is worth mentioning that Sobolev embedding is not compact on domains of infinite volume (see [17]). This leads to a major obstacle for proving the existence of attractors for PDEs on unbounded domains. For some deterministic equations, the difficulty caused by the unboundedness of domains can be overcome by the energy equation approach. The energy equation method was developed by Ball in [18] and used by many authors (see, for example, [19-22]). Under certain circumstances, the tail-estimates method can be used to deal with the problem caused by the unboundedness of domains (see $[11,23,24]$ ). In this article, we will use the idea of uniform estimates on the tail of solutions to study the existence of a random attractor of the stochastic reaction-diffusion equation with distribution derivatives and multiplicative noise on unbounded domain.

This article is organized as follows. In Section 2, we recall some basic concepts and properties for general random dynamics system. In Section 3, we provide some basic settings about Equation (1) and show that it generates a random dynamical system on $L^{2}\left(\mathbb{R}^{n}\right)$. In the last section, firstly we prove the uniform estimates of solutions which include the uniform estimates on the tails of solutions. Then establish the asymptotic compactness of the solution operator by given uniform estimates on the tails of solutions and prove the existence of a random attractor.

In the sequel, we use $\|\cdot\|_{p}$ be the norm of $L^{p}\left(\mathbb{R}^{n}\right)(p \geq 1),|v|$ the modular of $v, m(e)$, sometimes we also write it as $|e|$ the Lebesgue measure of $e \subset \mathbb{R}^{n}, \mathbb{R}^{n}(|v| \geq \tilde{M}) \triangleq\left\{x \in \mathbb{R}^{n}|| v(x) \mid \geq \tilde{M}\right\}$, and $C$ an arbitrary positive constant, which may be different from line to line and even in the same line, and $\|\cdot\|,(\cdot, \cdot)$ to denote the norm and inner product of $L^{2}\left(\mathbb{R}^{n}\right)$, respectively.

\section{Preliminaries and abstract results}

\subsection{Preliminaries}

As mentioned in the introduction, our main purpose is to prove the existence of the $\left(L^{2}\left(\mathbb{R}^{n}\right), L^{p}\left(\mathbb{R}^{n}\right)\right)$-random attractor. For that matter, first, we will recapitulate basic concepts related to random attractors for stochastic dynamical systems. The reader is referred to $[3,25]$ for more details.

Let $\left(X,\|\cdot\|_{X}\right)$ be separable Hilbert space with the Borel $\sigma$-algebra $\mathcal{B}(X)$. Let $(\Omega, \mathcal{F}, P)$ be a probability space.

Definition 1. $\left(\Omega, \mathcal{F}, P,\left(\vartheta_{t}\right)_{t \in \mathbb{R}}\right)$ is called a metric dynamical system if $\vartheta: \mathbb{R} \times \Omega \rightarrow \Omega$ is $(\mathcal{B}(\mathbb{R}) \times$ $\mathcal{F}, \mathcal{F}$ )-measurable, $\vartheta_{0}$ is the identity on $\Omega, \vartheta_{s+t}=\vartheta_{t} \circ \vartheta_{s}$ for all $s, t \in \mathbb{R}$ and $\vartheta_{t} P=P$ for all $t \in \mathbb{R}$. 
Definition 2. A continuous random dynamical system (RDS) on $X$ over a metric dynamical system $\left(\Omega, \mathcal{F}, P,\left(\vartheta_{t}\right)_{t \in \mathbb{R}}\right)$ is a mapping

$$
\phi: \mathbb{R}^{+} \times \Omega \times X \longrightarrow X, \quad(t, \omega, x) \mapsto \phi(t, \omega, x),
$$

which is $\left(\mathcal{B}\left(\mathbb{R}^{+}\right) \times \mathcal{F} \times \mathcal{B}(X), \mathcal{B}(X)\right)$ - measurable and satisfies, for $\mathbb{P}$-a.e. $\omega \in \Omega$.

1. $\phi(0, \omega, \cdot)$ is the identity on $X$,

2. $\phi(t+s, \omega, \cdot)=\phi\left(t, \vartheta_{s} \omega, \cdot\right) \circ \phi(s, \omega, \cdot)$ for all $t, s \in \mathbb{R}^{+}$,

3. $\phi(t, \omega, \cdot): X \rightarrow X$ is continuous for all $t \in \mathbb{R}^{+}$.

Hereafter, we always assume that $\phi$ is continuous $\operatorname{RDS}$ on $\mathrm{X}$ over $\left(\Omega, \mathcal{F}, P,\left(\vartheta_{t}\right)_{t \in \mathbb{R}}\right)$.

Definition 3. A set-valued mapping $\{D(\omega)\}: \Omega \rightarrow 2^{X}, \omega \rightarrow D(\omega)$, is said to be a random set if the mapping $\omega \mapsto d(u, D(\omega))$ is measurable for every $u \in X$. If $D(\omega)$ is also closed (compact) for each $\omega \in \Omega,\{D(\omega)\}$ is called a random closed(compact) set. A random set $\{D(\omega)\}$ is said to be bounded if there exist $u_{0} \in X$ and a random variable $R_{1}(\omega)>0$ such that $D(\omega) \subset\left\{u \in X:\left\|u-u_{0}\right\|_{X} \leq R_{1}(\omega)\right\}$ for all $\omega \in \Omega$.

Definition 4. A random bounded set $\{D(\omega)\}$ is called tempered provided for $P$-a.e, $\omega \in \Omega$, $\lim _{t \rightarrow+\infty} e^{-\beta t} d\left(D\left(\vartheta_{-t} \omega\right)\right)=0$ for all $\beta>0$, where $d(D)=\sup \left\{\|b\|_{X}: b \in D\right\}$.

Definition 5. Let $\mathfrak{D}$ be a collection of random subset of $X$ and $\{K(\omega)\} \in \mathfrak{D}$. Then $\{K(\omega)\}$ is called a random absorbing set for $\phi$ in $\mathfrak{D}$ for every $D \in \mathfrak{D}$ and $P$-a.e, $\omega \in \Omega$, there exist $t_{0}(\omega)$ such that $\phi\left(t, \vartheta_{-t} \omega, D\left(\vartheta_{-t} \omega\right)\right) \subseteq$ $K(\omega)$ for all $t \geq t_{0}(\omega)$.

Definition 6. A random set $\left\{K_{1}(\omega)\right\}$ is said to be a random attracting set if for every tempered random set $\{D(\omega)\}$, and $P$-a.e, $\omega \in \Omega$, we have

$$
\lim _{t \rightarrow+\infty} d_{H}\left(\phi\left(t, \vartheta_{-t} \omega, D\left(\vartheta_{-t} \omega\right), K_{1}(\omega)\right)\right)=0
$$

where $d_{H}$ is the Hausdorff semi-distance given by $d_{H}(E, F)=\sup _{u \in E} \inf _{v \in F}\|u-v\|_{X}$ for every $E, F \subset X$.

Definition 7. Let $\mathfrak{D}$ be the set of all random tempered sets in $X$. Then $\phi$ is said to be asymptotically compact in $X$ if for $P$-a.e. $\omega \in \Omega,\left\{\phi\left(t_{n}, \vartheta_{-t_{n}} \omega, X_{n}\right)\right\}_{n=1}^{\infty}$ has a convergent subsequence in $X$ whenever $t_{n} \rightarrow \infty$, and $X_{n} \in B\left(\vartheta_{-t_{n}} \omega\right)$ with $\{B(\omega)\} \in \mathfrak{D}$.

Definition 8. A random compact set $\{\mathcal{A}(\omega)\}$ is said to be a random attractor if it is andom attracting set and $\phi(t, \omega, \mathcal{A}(\omega))=\mathcal{A}\left(\vartheta_{-t} \omega\right)$ for $P$-a.e. $\omega \in \Omega$ and all $t \geq 0$.

Definition 9. Let $\phi$ be a continuous random dynamical system on $X$ over $\left(\Omega, \mathcal{F}, P,\left(\vartheta_{t}\right)_{t \in \mathbb{R}}\right)$. If there is a closed random tempered absorbing set $\{K(\omega)\}$ of $\phi$ and $\phi$ is asymptotically compact in $X$, then $\{\mathcal{A}(\omega)\}$ is a random attractor of $\phi$, where

$$
\mathcal{A}(\omega)=\bigcap_{t>0} \overline{\bigcup_{\tau \geq t} \phi\left(\tau, \vartheta_{-\tau} \omega, K\left(\vartheta_{-\tau} \omega\right)\right)}, \omega \in \Omega
$$

Moreover, $\{\mathcal{A}(\omega)\}$ is the unique attractor of $\phi$.

Theorem 1. [13] Let $X$ be a Banach space and $\{\phi(t)\}_{t \geq 0}$ be a continuous random dynamical system on $X$. Then $\{\phi(t)\}_{t \geq 0}$ has a random attractor in $X$ provided that the following conditions hold:

1. $\{\phi(t)\}_{t \geq 0}$ has a bounded absorbing set in $X$.

2. For any bounded subset $B(\omega)$ of $X$, we have $\kappa(\phi(t) B(\omega)) \rightarrow 0$ as $t \rightarrow \infty$.

\subsection{Abstract results}

In the following, we will give some simple properties of bounded sets in $L^{p}\left(\mathbb{R}^{n}\right)$, which are useful for establishing the asymptotic compactness of the random dynamical system in $L^{p}\left(\mathbb{R}^{n}\right)$. 
Lemma 1. $[26,27]$ Suppose a RDS $\phi$ has a bounded absorbing set $B(\omega)$ in $L^{p}(\Omega)$. Then for P-a.s. $\omega \in \Omega, \zeta>0$ and bounded $B \subset L^{p}(\Omega)$, there exist $\tilde{T}=\tilde{T}(B, \omega)$ and $\tilde{M}=\tilde{M}(\zeta, \omega)$ such that

$$
m\left(\Omega\left(\left|\phi\left(t, \vartheta_{-t} \omega\right) u\right| \geq \tilde{M}\right)\right)<\zeta \text { for any } u \in B \text { and } t \geq T .
$$

Lemma 2. $[13,28]$ Let $B$ be a bounded subset in $L^{p}(\Omega)(p \geq 1)$ and $\zeta>0$ be arbitrary. Assume that there is an $\tilde{M}=\tilde{M}(B, \zeta)$ such that for every $v \in B, \int_{\Omega(|v| \geq \tilde{M})}(|v|-\tilde{M})^{P} \leq \zeta$, then we have

$$
\int_{\Omega(|v| \geq 2 \tilde{M})}|v|^{p} \leq 2^{p+1} \zeta \text { for all } v \in B
$$

Lemma 3. [13] Let $B \subset L^{2}\left(\mathbb{R}^{n}\right) \cap L^{p}\left(\mathbb{R}^{n}\right)(p \geq 2)$ and be bounded in both $L^{2}\left(\mathbb{R}^{n}\right)$ and $L^{p}\left(\mathbb{R}^{n}\right)$. Then for every $\zeta>0, B$ has a finite $\zeta$-net in $L^{p}\left(\mathbb{R}^{n}\right)$ if there exists a positive constant $\tilde{M}=\tilde{M}(\zeta, \omega)$ which depends on $\zeta$, such that

(i) B has a finite $(3 \tilde{M})^{(2-p) / 2}(\zeta / 2)^{p / 2}-$ net in $L^{2}\left(\mathbb{R}^{n}\right)$.

(ii) $\left(\int_{\mathbb{R}^{n}(|v| \geq \tilde{M})}|v|^{p} d x\right)^{1 / p}<2^{-(2 p+2) / p} \zeta$ for every $v \in B$.

Motivated by Theorem 1, we will provide a simple and convenient criterion for the existence of random attractors in $\mathbb{R}^{n}$, which will be used later:

Corollary 1. $[13,24,29]$ Let $\{\phi(t)\}_{t \geq 0}$ be a continuous random dynamical system on $L^{2}\left(\mathbb{R}^{n}\right)$. Then $\{\phi(t)\}_{t \geq 0}$ has a $\left(L^{2}\left(\mathbb{R}^{n}\right), L^{2}\left(\mathbb{R}^{n}\right)\right)$-random attractors if and only if

1. $\{\phi(t)\}_{t \geq 0}$ has a $\left(L^{2}\left(\mathbb{R}^{n}\right), L^{2}\left(\mathbb{R}^{n}\right)\right)$-bounded absorbing set.

2. For any $\zeta>0$, there exist $R_{\zeta}>0$ and $T_{\zeta}>0$, such that $\int_{\mathbb{R}^{n} \backslash B(\omega)(0, R)}\left|\phi(t) v_{0}\right|^{2} d x<\zeta$ for any $v_{0} \in B_{0}$, provided that $t \geq T_{\zeta}$ and $R \geq R_{\zeta}$.

3. For any $R>0$ and any bounded set $B \subset Y$, we have $\kappa\left(\left.\phi(t) B\right|_{B(0, R)}\right) \rightarrow 0$ as $t \rightarrow \infty$, where $B(0, R)=\{x \in$ $\left.\mathbb{R}^{n}:|x|<R\right\},\left.\phi(t) B\right|_{B(0, R)}=\left\{\left.\phi(t) v_{0}\right|_{B(0, R)}: v_{0} \in B\right\}$ and $\left.v(x)\right|_{B(0, R)}=\left\{\begin{array}{l}v(x) \text { if }|x|<R, \\ 0 \text { others wise. }\end{array}\right.$

As mentioned ahead, it is very hard to obtain the continuity of the random dynamical system in $L^{p}\left(\mathbb{R}^{n}\right)$, and the norm-to-weak continuity is also not available since there is no nested relation between $L^{2}\left(\mathbb{R}^{n}\right)$ and $L^{p}\left(\mathbb{R}^{n}\right)$ for $p \neq 2$. So it is very difficult to obtain the invariance of the random attractor by the continuity or norm-to-weak continuity of the random dynamical system. In this paper, we will establish the invariance of the $\left(L^{2}\left(\mathbb{R}^{n}\right), L^{p}\left(\mathbb{R}^{n}\right)\right)$-random attractor by the continuity of $\{\phi(t)\}_{t \geq 0}$ in $L^{2}\left(\mathbb{R}^{n}\right)$ and the asymptotic compactness in $L^{p}\left(\mathbb{R}^{n}\right)$, see Theorem 2. For that matter, we give the convenient lemma as following.

Lemma 4. [13] Let $X$ and $Y$ be two Banach spaces with norm $\|\cdot\|_{X}$ and $\|\cdot\|_{Y}$, respectively, $B \subset \mathfrak{D}(X) \cap \mathfrak{D}(Y)$. Assume that $\left\{x_{n}\right\}_{n=1}^{\infty} \subset B$ and $x_{n} \stackrel{\|\cdot\|_{X}}{\longrightarrow} x_{0}, x_{n} \stackrel{\|\cdot\|_{X}}{\longrightarrow} y_{0}, x_{0}, y_{0} \in B(\omega)$. Then $x_{0}=y_{0}$, where $\mathfrak{D}(X)$ denotes the collection of all bounded subsets of $X$.

Theorem 2. [27] Let $\phi(t, \omega)$ be a quasi-continuous random dynamical system on $L^{p}\left(\mathbb{R}^{n}\right)$ over an ergodic system $\left(\Omega, \mathcal{F}, P ; \vartheta_{t}\right)$. suppose that $\phi$ has a $\left(L^{2}\left(\mathbb{R}^{n}\right), L^{2}\left(\mathbb{R}^{n}\right)\right)$-random attractor. Then $\phi$ has a $\left(L^{2}\left(\mathbb{R}^{n}\right), L^{p}\left(\mathbb{R}^{n}\right)\right)$-random attractor provided that the following condition hold:

1. $\phi$ has a $\left(L^{2}\left(\mathbb{R}^{n}\right), L^{p}\left(\mathbb{R}^{n}\right)\right)$-bounded absorbing set $B_{0}(\omega)$,

2. $\phi$ is $\left(L^{2}\left(\mathbb{R}^{n}\right), L^{p}\left(\mathbb{R}^{n}\right)\right)$-omega-limit compact, that is for any bounded non-random set $B \subset L^{p}\left(\mathbb{R}^{n}\right)$, we have

$$
\lim _{T \rightarrow \infty} \kappa\left(\bigcup_{t \geq T} \phi\left(t, \vartheta_{-t} \omega\right) B\right)=0, \quad P \text {-a.s. } \omega \in \Omega
$$

Under the assumptions in Theorem 2, we will prove one of the random attractors is

$$
\mathcal{A}(\omega)=\bigcup_{B \subset L^{p}\left(\mathbb{R}^{n}\right)} A(B, \omega)
$$


where the union is taken over all bounded subsets of $L^{p}\left(\mathbb{R}^{n}\right)$, and $A(B, \omega)$ is the weakly sequence omega-limit set, that is,

$$
A(B, \omega)=\bigcap_{T \geq 0} \overline{\bigcup_{t \geq T} \phi\left(t, \vartheta_{-t} \omega\right) B^{W S}}
$$

for any bounded set $B \subset L^{p}\left(\mathbb{R}^{n}\right)$.

Using the same method as in [28], one can prove that if $\phi$ is omega-limit compact then $A(B, \omega)$ has the following character:

$$
x \in A(B, \omega) \text { iff } \exists x_{n} \in B, t_{n} \rightarrow \infty \text { such that } \phi\left(t_{n}, \vartheta_{-t_{n}} \omega\right) x_{n} \rightarrow x .
$$

Remark 1. $[13,27]$ From the proof of the Theorem 2, obviously, we have that the $\left(L^{2}\left(\mathbb{R}^{n}\right), L^{2}\left(\mathbb{R}^{n}\right)\right)$-random attractor coincides with the $\left(L^{2}\left(\mathbb{R}^{n}\right), L^{p}\left(\mathbb{R}^{n}\right)\right)$-random attractor.

Secondly, going by the idea of [30], we give a criterion or detailed scheme for verifying the existence of the $\left(L^{2}\left(\mathbb{R}^{n}\right), L^{p}\left(\mathbb{R}^{n}\right)\right)$-random attractor, which is very useful in our later discussion.

Theorem 3. Let $\{\phi(t)\}_{t \geq 0}$ be a continuous random dynamical system on $L^{2}\left(\mathbb{R}^{n}\right)$ and a random dynamical system on $L^{p}\left(\mathbb{R}^{n}\right)$, where $2 \leq p<\infty$. suppose that $\{\phi(t)\}_{t \geq 0}$ has a $\left(L^{2}\left(\mathbb{R}^{n}\right), L^{2}\left(\mathbb{R}^{n}\right)\right)$-random attractor. Then $\{\phi(t)\}_{t \geq 0}$ has a $\left(L^{2}\left(\mathbb{R}^{n}\right), L^{p}\left(\mathbb{R}^{n}\right)\right)$-random attractor provided that the following condition hold:

1. $\{\phi(t)\}_{t \geq 0}$ has a $\left(L^{2}\left(\mathbb{R}^{n}\right), L^{p}\left(\mathbb{R}^{n}\right)\right)$-bounded absorbing set $B_{0}(\omega)$,

2. For any $\zeta>0$ and any bounded (with respect to $\|\cdot\|_{2}$ ) subset $B$, there exist positive constants $\tilde{M}=$ $\tilde{M}(\zeta, B(\omega), \omega)$ and $\tilde{T}=\tilde{T}(\zeta, B(\omega), \omega)$, such that

$$
\int_{\mathbb{R}^{n}\left(\left|\phi(t) v_{0}\right| \geq \tilde{M}\right)}\left|\phi(t) v_{0}\right|^{p}<2^{-(2 p+2) / \zeta^{p}} \text { for any } v_{0} \in B(\omega) \text { and } t \geq \tilde{T} .
$$

Proof. Using Theorem 2, we only need to verify that $\{\phi(t)\}_{t \geq 0}$ is $\left(L^{2}\left(\mathbb{R}^{n}\right), L^{p}\left(\mathbb{R}^{n}\right)\right)$-asymptotically compact. As in Theorem 2, denote the $\left(L^{2}\left(\mathbb{R}^{n}\right), L^{2}\left(\mathbb{R}^{n}\right)\right)$-bounded absorbing set by $B_{2}(\omega)$ and let $B_{p}(\omega)=B_{0}(\omega) \cap$ $B_{2}(\omega)$. Then it is sufficient to prove that

$$
\text { for any } x_{n} \in B_{p}(\omega), t_{n} \rightarrow \infty,\left\{\phi\left(t_{n}\right) x_{n}\right\}_{n=1}^{\infty} \text { is precompact in } L^{p}\left(\mathbb{R}^{n}\right),
$$

which is equivalent to prove that for any $\zeta>0,\left\{\phi\left(t_{n}\right) x_{n}\right\}_{n=1}^{\infty}$ has a finite $\zeta$-net in $L^{p}\left(\mathbb{R}^{n}\right)$.

In fact, from the assumption that $\{\phi(t)\}_{t \geq 0}$ has a $\left(L^{2}\left(\mathbb{R}^{n}\right), L^{2}\left(\mathbb{R}^{n}\right)\right)$-random attractor, we know that there is a $\tilde{T}_{1}$ which depends on $\zeta$ and $\tilde{M}$, such that $\left\{\phi\left(t_{n}\right) x_{n} \mid t_{n} \geq \tilde{T}_{1}\right\}$ has a finite $(3 \tilde{M})^{(2-p) / 2}(\zeta /)^{p / 2}$-net in $L^{2}\left(\mathbb{R}^{n}\right)$. Taking $\tilde{T}_{0}=\max \left\{\tilde{T}_{1}, \tilde{T}\right\}$, then from Lemma 3, we know that $\left\{\phi\left(t_{n}\right) x_{n} \mid t_{n} \geq \tilde{T}_{0}\right\}$ has a finite $\zeta$-net in $L^{p}\left(\mathbb{R}^{n}\right)$. Since $t_{n} \rightarrow \infty$, we obtain that $\left\{\phi\left(t_{n}\right) x_{n}\right\}_{n=1}^{\infty}$ has a finite $\zeta$-net in $L^{p}\left(\mathbb{R}^{n}\right)$, too. Then from the arbitrariness of $\zeta$, we get that $\left\{\phi\left(t_{n}\right) x_{n}\right\}_{n=1}^{\infty}$ is precompact in $L^{p}\left(\mathbb{R}^{n}\right)$.

\section{The random dynamical system}

In this section, we show that there is a continuous random dynamical system generated by the stochastic reaction-diffusion equation defined on $\mathbb{R}^{n}$ with distribution derivatives and multiplicative noise:

$$
d u+(\lambda u-\Delta u) d t=\left(f(x)-g(u)+D_{j} f^{j}\right) d t+b u \circ d W(t), \text { in } \mathbb{R}^{+} \times \mathbb{R}^{n},
$$

with the initial value condition

$$
u(x, 0)=u_{0}(x), \quad x \in \mathbb{R}^{n},
$$

where $\lambda, b$ are a non-negative constant, $f^{j}, f \in L^{2}\left(\mathbb{R}^{n}\right), D_{j}=\frac{\partial}{\partial x_{j}}$ is distribution derivatives, and $g$ is a Lipschitz function which satisfies the conditions (3)-(5).

To model the random noise in Equation (12), we need to define a shift operator $\left\{\vartheta_{t}\right\}_{t \in \mathbb{R}}$ on $\Omega$ (where $\Omega$ is defined in the introduction) by

$$
\vartheta_{t} \omega(\cdot)=\omega(\cdot+t)-\omega(t), \quad t \in \mathbb{R},
$$

then $\left(\Omega, \mathcal{F}, \mathbb{P},\left\{\vartheta_{t}\right\}_{t \in \mathbb{R}}\right)$ is an ergodic metric dynamical system, see [3,25].

For our purpose, it is convenient to convert the Equation (12) into a deterministic system with a random parameter, and then show that it generates a random dynamical system. 
We now introduce an Ornstein-Uhlenbeck process given by the Brownian motion. Put

$$
z\left(\vartheta_{t} \omega\right):=-\int_{-\infty}^{0} e^{s}\left(\vartheta_{t} \omega\right)(s) d s, \quad t \in \mathbb{R},
$$

which is called the Ornstein-Uhlenbeck process and solves the Itô equation $d z+z d t=d W(t)$.

From $[3,11,31,32]$, it is known that the random variable $z(\omega)$ is tempered, and there is a $\vartheta_{t}$-invariant set $\tilde{\Omega} \subset \Omega$ of full $\mathbb{P}$ measure such that for every $\omega \in \tilde{\Omega}, t \mapsto z\left(\vartheta_{t} \omega\right)$ is continuous in $\mathrm{t} ; \lim _{t \rightarrow \pm \infty} \frac{\left|z\left(\vartheta_{t} \omega\right)\right|}{|t|}=0$; and $\lim _{t \rightarrow \pm \infty} \frac{1}{t} \int_{0}^{t} z\left(\vartheta_{s} \omega\right) d s=0$.

To show that Equation 12 generates a random dynamical system, we let

$$
v(t)=e^{-b z\left(\vartheta_{t} \omega\right)} u(t)
$$

where $u$ is a solution of Equation (12). Then we can consider the following evolution equation with random coefficients but without white noise:

$$
\frac{d v}{d t}+\lambda v-\Delta v=e^{-b z\left(\vartheta_{t} \omega\right)}\left(f(x)-g\left(e^{b z\left(\vartheta_{t} \omega\right)} v\right)+D_{j} f^{j}\right)+b z\left(\vartheta_{t} \omega\right) v,
$$

with the initial value condition

$$
v(x, 0)=v_{0}(x)=e^{-b z\left(\vartheta_{t} \omega\right)} u_{0}(x), \quad x \in \mathbb{R}^{n}
$$

We will consider Equations (16) and (17) for $\omega \in \tilde{\Omega}$ and write $\tilde{\Omega}$ as $\Omega$ from now on. By using the Galerkin method we can show that if $g$ satisfies (3)-(5), then for every $v_{0}$ in $L^{2}\left(\mathbb{R}^{n}\right)$, Equation (16) possesses a unique weak solution

$$
v\left(t, \omega, v_{0}\right) \in L^{\infty}\left(0, T, L^{2}\left(\mathbb{R}^{n}\right)\right) \bigcap L^{2}\left(0, T, H^{1}\left(\mathbb{R}^{n}\right)\right) \bigcap L^{p}\left(0, T, L^{p}\left(\mathbb{R}^{n}\right)\right) T>0
$$

which is continuous with respect to $v_{0}$ in $\in L^{2}\left(\mathbb{R}^{n}\right)$ for all $t>0$. Then equation (16) generates a continuous random dynamical system $\{\phi(t)\}_{t \geq 0}$ over $\left(\Omega, \mathcal{F}, \mathbb{P},\left\{\vartheta_{t}\right\}_{t \in \mathbb{R}}\right)$, where

$$
\phi\left(t, \omega, v_{0}\right)=v\left(t, \omega, v_{0}\right), \text { for } v_{0} \in L^{2}\left(\mathbb{R}^{n}\right), t \geq 0 \text { and for all } \omega \in \Omega .
$$

We define mapping $\varphi: \mathbb{R}^{+} \times \Omega \times L^{2}\left(\mathbb{R}^{n}\right) \rightarrow L^{2}\left(\mathbb{R}^{n}\right)$ by

$$
\varphi\left(t, \omega, u_{0}\right)=u\left(t, \omega, u_{0}\right)=e^{b z\left(\vartheta_{t} \omega\right)} \phi\left(t, \omega, v_{0}\right),
$$

for $v_{0} \in L^{2}\left(\mathbb{R}^{n}\right), t \geq 0$ and for all $\omega \in \Omega$.

Then $\varphi$ is a continuous random dynamical system associated with the Equation (12) on $L^{2}\left(\mathbb{R}^{n}\right)$. Note that the two random dynamical system are equivalent. It is easy to check that $\varphi$ has a random attractor provided $\phi$ possesses a random attractor. Then, we only need to consider the random dynamical system $\phi$.

\section{Existence of random attractors}

In this section, we prove the existence of a global random attractor for the random dynamical system $\phi$ associated with the semilinear reaction-diffusion Equation (12)-(13) on $\mathbb{R}^{n}$. The main result of this section can now be stated as follows. Firstly, we derive uniformly a priori estimates on the solutions of Equations (12)-(13) defined on $\mathbb{R}^{n}$ when $t \rightarrow \infty$ with the purpose of proving the existence of a bounded random absorbing set and the asymptotic compactness of the random dynamical system associated with the equation. In particular, we will show that the tails of the solutions for large space variable are uniformly small when time is sufficiently large.

From now on, we always assume that $\mathfrak{D}$ is the collection of all tempered random subsets of $L^{2}\left(\mathbb{R}^{n}\right), L^{p}\left(\mathbb{R}^{n}\right)$ with respect to $\left(\Omega, \mathcal{F}, \mathbb{P},\left\{\vartheta_{t}\right\}_{t \in \mathbb{R}}\right)$. The next lemma shows that $\phi$ has a random absorbing set in $\mathfrak{D}$. 
Lemma 5. Assume that $f^{j}, f \in L^{2}\left(\mathbb{R}^{n}\right)$, and (1.3)-(1.5) hold. Then there exists a random ball $\{K(\omega)\} \in \mathfrak{D}$ centered at 0 with random radius $\rho(\omega)>0$ such that $\{K(\omega)\}$ is a random absorbing set for $\phi$ in $\mathfrak{D}$, that is, for any $\{B(\omega)\} \in \mathfrak{D}$ and $\mathbb{P}$-a.e. $\omega \in \Omega$, there is $T_{B}(\omega)>0$ such that

$$
\phi\left(t, \vartheta_{-t} \omega, B\left(\vartheta_{-t} \omega,\right)\right) \subseteq K(\omega) \text { for all } t>T_{B}(\omega) .
$$

Proof. Taking the inner product of Equation (16) with $v$ in $L^{2}\left(\mathbb{R}^{n}\right)$, we have

$$
\frac{1}{2} \frac{d}{d t}\|v\|^{2}+\lambda\|v\|^{2}+\|\nabla v\|^{2}=-e^{-b z\left(\vartheta_{t} \omega\right)} \int_{\mathbb{R}^{n}} g\left(e^{b z\left(\vartheta_{t} \omega\right)} v\right) v d x+e^{-b z\left(\vartheta_{t} \omega\right)}\left((f, v)+\left(D_{j} f^{j}, v\right)\right)+b z\left(\vartheta_{t} \omega\right)\|v\|^{2} .
$$

By the condition (4), we obtain that

$$
e^{-b z\left(\vartheta_{t} \omega\right)} \int_{\mathbb{R}^{n}} g\left(e^{b z\left(\vartheta_{t} \omega\right)} v\right) v d x=e^{-2 b z\left(\vartheta_{t} \omega\right)} \int_{\mathbb{R}^{n}} g(u) u d x \geq \beta_{2} e^{b(p-2) z\left(\vartheta_{t} \omega\right)}\|v\|^{p}-\delta_{2}\|v\|^{2} .
$$

By the Hölder' inequality and the Young inequality, we conclude

$$
\begin{gathered}
e^{-b z\left(\vartheta_{t} \omega\right)}(f, v) \leq e^{-b z\left(\vartheta_{t} \omega\right)}\|f\| \cdot\|v\| \leq \frac{1}{2 \delta_{0}} e^{-2 b z\left(\vartheta_{t} \omega\right)}\|f\|^{2}+\frac{\delta_{0}}{2}\|v\|^{2} \\
e^{-b z\left(\vartheta_{t} \omega\right)}\left(D_{j} f^{j}, v\right)=e^{-b z\left(\vartheta_{t} \omega\right)}(\tilde{f}, \nabla v) \leq e^{-b z\left(\vartheta_{t} \omega\right)}\|\tilde{f}\| \cdot\|\nabla v\| \leq \frac{1}{2} e^{-2 b z\left(\vartheta_{t} \omega\right)}\|\tilde{f}\|^{2}+\frac{1}{2}\|\nabla v\|^{2},
\end{gathered}
$$

where $\tilde{f}=\left(f^{1}, \ldots, f^{n}\right)$ and $\|\tilde{f}\|^{2}=\sum_{j=1}^{n}\left|f^{j}\right|^{2}$.

Then substituting Equations (20)-(22) into (19), it yields

$$
\frac{d}{d t}\|v\|^{2}-\left(2 b z\left(\vartheta_{t} \omega\right)-\delta_{0}\right)\|v\|^{2}+\|\nabla v\|^{2}+2 \beta_{2} e^{b(p-2) z\left(\vartheta_{t} \omega\right)}\|v\|^{p} \leq \frac{1}{\delta_{0}} e^{-2 b z\left(\vartheta_{t} \omega\right)}\left(\|f\|^{2}+\delta_{0}\|\tilde{f}\|^{2}\right),
$$

where $\delta_{0}=\lambda-\delta_{2}$, By the condition (5), $\delta_{0}$ is non-negative constant. Hence, we can rewrite (23) as

$$
\frac{d}{d t}\|v\|^{2}-\left(2 b z\left(\vartheta_{t} \omega\right)-\delta_{0}\right)\|v\|^{2} \leq \frac{1}{\delta_{0}} e^{-2 b z\left(\vartheta_{t} \omega\right)}\left(\|f\|^{2}+\delta_{0}\|\tilde{f}\|^{2}\right) .
$$

By applying the Gronwall's Lemma to (24), we find that

$$
\begin{aligned}
& \left\|v\left(t, \omega, v_{0}(\omega)\right)\right\|^{2} \\
& \leq e^{2 \int_{0}^{t} b z\left(\vartheta_{s} \omega\right) d s-\delta_{0} t}\left\|v_{0}(\omega)\right\|^{2}+\frac{\|f\|^{2}+\delta_{0}\|\tilde{f}\|^{2}}{\delta_{0}} e^{2 b \int_{0}^{t} z\left(\vartheta_{s} \omega\right) d s-\delta_{0} t} \int_{0}^{t} e^{-2 b z\left(\vartheta_{s} \omega\right)-2 b \int_{0}^{s} z\left(\vartheta_{\tau} \omega\right) d \tau+\delta_{0} s} d s .
\end{aligned}
$$

By replacing $\omega$ by $\vartheta_{-t} \omega$ in (25), we get

$$
\begin{aligned}
& \left\|v\left(t, \vartheta_{-t} \omega, v_{0}\left(\vartheta_{-t} \omega\right)\right)\right\|^{2} \\
& \leq e^{2 b \int_{0}^{t} z\left(\vartheta_{s-t} \omega\right) d s-\delta_{0} t}\left\|v_{0}\left(\vartheta_{-t} \omega\right)\right\|^{2}+\frac{\|f\|^{2}+\delta_{0}\|\tilde{f}\|^{2}}{\delta_{0}} e^{2 b \int_{0}^{t} z\left(\vartheta_{s-t} \omega\right) d s-\delta_{0} t} \int_{0}^{t} e^{-2 b z\left(\vartheta_{s-t} \omega\right)-2 b \int_{0}^{s} z\left(\vartheta_{\tau-t} \omega\right) d \tau+\delta_{0} s} d s \\
& \leq e^{2 b \int_{-t}^{0} z\left(\vartheta_{s} \omega\right) d s-\delta_{0} t}\left\|v_{0}\left(\vartheta_{-t} \omega\right)\right\|^{2}+\frac{\|f\|^{2}+\delta_{0}\|\tilde{f}\|^{2}}{\delta_{0}} \int_{-t}^{0} e^{-2 b z\left(\vartheta_{s} \omega\right)+2 b \int_{s}^{0} z\left(\vartheta_{\tau} \omega\right) d \tau+\delta_{0} s} d s \\
& \leq e^{2 b \int_{-t}^{0} z\left(\vartheta_{s} \omega\right) d s-\delta_{0} t}\left\|v_{0}\left(\vartheta_{-t} \omega\right)\right\|^{2}+\frac{\|f\|^{2}+\delta_{0}\|\tilde{f}\|^{2}}{\delta_{0}} \int_{-\infty}^{0} e^{-2 b z\left(\vartheta_{s} \omega\right)+2 b \int_{s}^{0} z\left(\vartheta_{\tau} \omega\right) d \tau+\delta_{0} s} d s
\end{aligned}
$$

By the properties of Ornstein-Uhlenbeck process,

$$
\int_{-\infty}^{0} e^{-2 b z\left(\vartheta_{s} \omega\right)+2 b \int_{s}^{0} z\left(\vartheta_{\tau} \omega\right) d \tau+\delta_{0} s} d s<+\infty .
$$

Notice that $\{B(\omega)\} \in \mathfrak{D}$ is tempered, then for any $v_{0}\left(\vartheta_{-t} \omega\right) \in B\left(\vartheta_{-t} \omega\right)$,

$$
\lim _{t \rightarrow+\infty} e^{2 b \int_{-t}^{0} z\left(\vartheta_{s} \omega\right) d s-\delta_{0} t}\left\|v_{0}\left(\vartheta_{-t} \omega\right)\right\|^{2}=0 .
$$


We can choose

$$
\rho(\omega)=1+\frac{\|f\|^{2}+\delta_{0}\|\tilde{f}\|^{2}}{\delta_{0}} \int_{-\infty}^{0} e^{-2 b z\left(\vartheta_{s} \omega\right)+2 b \int_{s}^{0} z\left(\vartheta_{\tau} \omega\right) d \tau+\delta_{0} s} d s
$$

and let

$$
K(\omega)=\left\{u \in L^{2}\left(\mathbb{R}^{n}\right):\|u\|^{2} \leq \rho(\omega)\right\} .
$$

Then $\{K(\omega)\} \in \mathfrak{D}$, and $\{K(\omega)\}$ is a random absorbing set for $\phi$ in $\mathfrak{D}$, which completes the proof.

Lemma 6. Assume that $f^{j}, f \in L^{2}\left(\mathbb{R}^{n}\right)$, and Equations (3)-(5) hold. The random dynamical system $\{\phi(t)\}_{t \geq 0}$ has a $\left(L^{2}\left(\mathbb{R}^{n}\right), L^{p}\left(\mathbb{R}^{n}\right)\right)$ and $\left(L^{2}\left(\mathbb{R}^{n}\right), H^{1}\left(\mathbb{R}^{n}\right)\right)$-bounded absorbing set, that is, there exists a random radius $\tilde{\rho}(\omega)$ such that for any $\{B(\omega)\} \in \mathfrak{D}$ and $v_{0}(\omega) \in B(\omega)$, there exists a $T_{B}(\omega)>0$ such that the solution $\phi$ of (27) satisfies for $\mathbb{P}$-a.e. $\omega \in \Omega$, for all $t \geq T_{B}(\omega)$,

$$
\left\|\phi\left(t, \vartheta_{-t} \omega, v_{0}\left(\vartheta_{-t} \omega\right)\right)\right\|^{p}+\left\|\phi\left(t, \vartheta_{-t} \omega, v_{0}\left(\vartheta_{-t} \omega\right)\right)\right\|^{2}+\left\|\nabla \phi\left(t, \vartheta_{-t} \omega, v_{0}\left(\vartheta_{-t} \omega\right)\right)\right\|^{2} \leq \tilde{\rho}(\omega) .
$$

Proof. Taking the inner product of Equation (16) with $v$ in $L^{2}\left(\mathbb{R}^{n}\right)$, we have

$$
\frac{1}{2} \frac{d}{d t}\|v\|^{2}+\lambda\|v\|^{2}+\|\nabla v\|^{2}=-e^{-b z\left(\vartheta_{t} \omega\right)} \int_{\mathbb{R}^{n}} g\left(e^{b z\left(\vartheta_{t} \omega\right)} v\right) v d x+e^{-b z\left(\vartheta_{t} \omega\right)}\left((f, v)+\left(D_{j} f^{j}, v\right)\right)+b z\left(\vartheta_{t} \omega\right)\|v\|^{2} .
$$

Using Equations (20)-(21) and Lemma 5, we conclude from (31) that

$\frac{1}{2} \frac{d}{d t}\|v\|^{2}+\frac{\delta_{0}}{2}\|v\|^{2}+\frac{1}{2}\|\nabla v\|^{2}+\beta_{2} e^{b(p-2) z\left(\vartheta_{t} \omega\right)}\|v\|^{p} \leq \frac{1}{2 \delta_{0}} e^{-2 b z\left(\vartheta_{t} \omega\right)}\|f\|^{2}+b z\left(\vartheta_{t} \omega\right) \rho(\omega)+e^{-b z\left(\vartheta_{t} \omega\right)}\left(D_{j} f^{j}, v\right)$.

Hence, we can rewrite Equation (32) as

$$
\frac{1}{2} \frac{d}{d t}\|v\|^{2}+\frac{\delta_{0}}{2}\|v\|^{2}+\frac{1}{2}\|\nabla v\|^{2}+\beta_{2} e^{b(p-2) z\left(\vartheta_{t} \omega\right)}\|v\|^{p} \leq \tilde{\rho}(\omega)+e^{-b z\left(\vartheta_{t} \omega\right)}\left(D_{j} f^{j}, v\right) .
$$

Noticing that

$$
\|\nabla v+\tilde{f}\|^{2} \leq 2\|\nabla v\|^{2}+2\|\tilde{f}\|^{2}
$$

by (34), we conclude from (33) that

$$
\frac{d}{d t}\|v\|^{2}+\mathcal{C}\left(\|\nabla v+\tilde{f}\|^{2}+\|v\|^{2}+\|v\|^{p}\right) \leq \tilde{\rho}(\omega) .
$$

Integrating the Equation (35) from $t$ to $t+1$, and using Lemma 5 , we can find a $T_{B}(\omega)>0$, such that for all $t \geq T_{B}(\omega)$,

$$
\int_{t}^{t+1}\left(\|\nabla v+\tilde{f}\|^{2}+\|v\|^{2}+\|v\|^{p}\right) \leq \tilde{\rho}(\omega)
$$

On the other hand , multiplying Equation (16) with $v_{t}$, and integrating over $\mathbb{R}^{n}$ we find that

$$
\left\|v_{t}\right\|^{2}+\frac{1}{2} \frac{d}{d t}\left(\|\nabla v\|^{2}+2(\tilde{f}, \nabla v)+\lambda\|v\|^{2}+2 \int_{\mathbb{R}^{n}} g(u) d x\right)=e^{-b z\left(\vartheta_{t} \omega\right)}\left(f, v_{t}\right)+\frac{1}{2} b\left|z\left(\vartheta_{t} \omega\right)\right|\|v\|^{2}+\frac{1}{2}\left\|v_{t}\right\|^{2} .
$$

By the Hölder's inequality and the Young's inequality, we conclude

$$
e^{-b z\left(\vartheta_{t} \omega\right)}\left(f, v_{t}\right) \leq e^{-b z\left(\vartheta_{t} \omega\right)}\|f\| \cdot\left\|v_{t}\right\| \leq \frac{1}{2} e^{-2 b z\left(\vartheta_{t} \omega\right)}\|f\|^{2}+\frac{1}{2}\left\|v_{t}\right\|^{2} .
$$

Now, let $G(s)=\int_{0}^{s} g(\tau) d \tau$; then by condition (4) again, we can conclude that

$$
\tilde{\beta}_{2}|s|^{p}-\tilde{\delta}_{2}|s|^{2} \leq G(s) \leq \tilde{\beta}_{1}|s|^{p}+\tilde{\delta}_{1}|s|^{2} .
$$

Therefore

$$
\tilde{\beta}_{2} \int_{R^{n}}|u|^{p} d x-\tilde{\delta}_{2} \int_{R^{n}}|u|^{2} d x \leq \int_{R^{n}} G(u) d x \leq \tilde{\beta}_{1} \int_{R^{n}}|u|^{p} d x+\tilde{\delta}_{1} \int_{R^{n}}|u|^{2} d x .
$$


Then inserting (38)-(39) into (37) and by Lemma 5, it yields

$$
\frac{d}{d t}\left(\|\nabla v\|^{2}+2(\tilde{f}, \nabla v)+\|\tilde{f}\|^{2}-\|\tilde{f}\|^{2}+\lambda\|v\|^{2}+\tilde{\beta}_{2} \int_{R^{n}}|u|^{p} d x-\tilde{\delta}_{2} \int_{R^{n}}|u|^{2} d x\right) \leq \tilde{\rho}(\omega) .
$$

Hence, we can rewrite (40) as

$$
\frac{d}{d t}\left(\|\nabla v+\tilde{f}\|^{2}+\|v\|^{2}+\|v\|^{p}\right) \leq \tilde{\rho}(\omega) .
$$

Combining with (36) and (41), by the uniform Gronwall's Lemma, we deduce that

$$
\|\nabla v+\tilde{f}\|^{2}+\|v\|^{2}+\|v\|^{p} \leq \tilde{\rho}(\omega) .
$$

Using $\|\nabla v\|^{2} \leq 2\|\nabla v+\tilde{f}\|^{2}+2\|\tilde{f}\|^{2}$ and Equation (42) implies that for $t \leq T_{B}(\omega)+1$,

$$
\|\nabla v\|^{2}+\|v\|^{2}+\|v\|^{p} \leq \tilde{\rho}(\omega) .
$$

The proof is concluded.

Lemma 7. Assume that $f^{j}, f \in L^{2}\left(\mathbb{R}^{n}\right)$, and (3)-(5) hold. Then there exists a tempered random variable $\tilde{R_{1}}(\omega)>0$ such that for any $\{B(\omega)\} \in \mathfrak{D}$ and $v_{0}(\omega) \in B(\omega)$, there exists a $T_{B}(\omega)>0$ such that the solution $\phi$ of $(16)$ satisfies for $\mathbb{P}$-a.e. $\omega \in \Omega$, for all $t \geq T_{B}(\omega)$,

$$
\int_{t}^{t+1}\left\|\nabla \phi\left(s, \vartheta_{-t-1} \omega, v_{0}\left(\vartheta_{-t-1} \omega\right)\right)\right\|^{2} d s \leq \tilde{R_{1}}(\omega) .
$$

Proof. By substituting $t$ by $\hat{T}$ and $\omega$ by $\vartheta_{-t} \omega$ in (25) for any $\hat{T} \geq 0$, we find that

$$
\begin{aligned}
& \left\|v\left(\hat{T}, \vartheta_{-t} \omega, v_{0}\left(\vartheta_{-t} \omega\right)\right)\right\|^{2} \\
& \leq e^{2 b \int_{0}^{\hat{T}} z\left(\vartheta_{s-t} \omega\right) d s-\delta_{0} \hat{T}}\left\|v_{0}\left(\vartheta_{-t} \omega\right)\right\|^{2}+\frac{\|f\|^{2}+\delta_{0}\|\tilde{f}\|^{2}}{\delta_{0}} e^{2 b \int_{0}^{\hat{T}} z\left(\vartheta_{s-t} \omega\right) d s-\delta_{0} \hat{T}} \int_{0}^{\hat{T}} e^{-2 b z\left(\vartheta_{s-t} \omega\right)-2 b \int_{0}^{s} z\left(\vartheta_{\tau-t} \omega\right) d \tau+\delta_{0} s} d s .
\end{aligned}
$$

Multiplying two sides of the equation (45) by $e^{2 b \int_{T}^{t} z\left(\vartheta_{\tau-t} \omega\right) d \tau-\delta_{0}(t-\hat{T})}$, then simplifying it, we find that for all $t \geq \hat{T}$

$$
\begin{aligned}
& e^{2 b \int_{\hat{T}}^{t} z\left(\vartheta_{\tau-t} \omega\right) d \tau-\delta_{0}(t-\hat{T})}\left\|v\left(\hat{T}, \vartheta_{-t} \omega, v_{0}\left(\vartheta_{-t} \omega\right)\right)\right\|^{2} \\
& \leq e^{2 b \int_{0}^{t} z\left(\vartheta_{s-t} \omega\right) d s-\delta_{0} t}\left\|v_{0}\left(\vartheta_{-t} \omega\right)\right\|^{2}+\frac{\|f\|^{2}+\delta_{0}\|\tilde{f}\|^{2}}{\delta_{0}} \int_{0}^{\hat{T}} e^{-2 b z\left(\vartheta_{s-t} \omega\right)+2 b \int_{s}^{t} z\left(\vartheta_{s-t} \omega\right) d s-\delta_{0}(t-s)} d s .
\end{aligned}
$$

By the Gronwall's Lemma, of the Equation (23) we get that for all $t \geq \hat{T}$,

$$
\begin{aligned}
\left\|v\left(t, \omega, v_{0}(\omega)\right)\right\|^{2} \leq & e^{2 b \int_{\hat{T}}^{t} z\left(\vartheta_{s} \omega\right) d s-\delta_{0}(t-\hat{T})}\left\|v\left(\hat{T}, \omega, v_{0}(\omega)\right)\right\|^{2}-\int_{\hat{T}}^{t} e^{2 b \int_{s}^{t} z\left(\vartheta_{\tau} \omega\right) d \tau+\delta_{0}(s-t)}\left\|\nabla v\left(s, \omega, v_{0}(\omega)\right)\right\|^{2} d s \\
& +\frac{\|f\|^{2}+\delta_{0}\|\tilde{f}\|^{2}}{\delta_{0}} \int_{\hat{T}}^{t} e^{-2 b z\left(\vartheta_{s} \omega\right)+2 b \int_{s}^{t} z\left(\vartheta_{\tau} \omega\right) d \tau+\delta_{0}(s-t)} d s
\end{aligned}
$$

which obviously gives

$$
\begin{aligned}
& \int_{\hat{T}}^{t} e^{2 b \int_{s}^{t} z\left(\vartheta_{\tau} \omega\right) d \tau+\delta_{0}(s-t)}\left\|\nabla v\left(s, \omega, v_{0}(\omega)\right)\right\|^{2} d s \\
& \leq e^{2 b \int_{\hat{T}}^{t} z\left(\vartheta_{s} \omega\right) d s-\delta_{0}(t-\hat{T})}\left\|v\left(\hat{T}, \omega, v_{0}(\omega)\right)\right\|^{2}+\frac{\|f\|^{2}+\delta_{0}\|\tilde{f}\|^{2}}{\delta_{0}} \int_{\hat{T}}^{t} e^{-2 b z\left(\vartheta_{s} \omega\right)+2 b \int_{s}^{t} z\left(\vartheta_{\tau} \omega\right) d \tau+\delta_{0}(s-t)} d s .
\end{aligned}
$$

By replacing $\omega$ by $\vartheta_{-t} \omega$ into (48), we get 


$$
\begin{aligned}
& \int_{\hat{T}}^{t} e^{2 b \int_{s}^{t} z\left(\vartheta_{\tau-t} \omega\right) d \tau+\delta_{0}(s-t)}\left\|\nabla v\left(s, \vartheta_{-t} \omega, v_{0}\left(\vartheta_{-t} \omega\right)\right)\right\|^{2} d s \\
& \leq e^{2 b \int_{\hat{T}}^{t} z\left(\vartheta_{s-t} \omega\right) d s-\delta_{0}(t-\hat{T})}\left\|v\left(\hat{T}, \vartheta_{-t} \omega, v_{0}\left(\vartheta_{-t} \omega\right)\right)\right\|^{2}+\frac{\|f\|^{2}+\delta_{0}\|\tilde{f}\|^{2}}{\delta_{0}} \int_{\hat{T}}^{t} e^{-2 b z\left(\vartheta_{s-t} \omega\right)+2 b \int_{s}^{t} z\left(\vartheta_{\tau-t} \omega\right) d \tau+\delta_{0}(s-t)} d s .
\end{aligned}
$$

By using (46) into (49), we have

$$
\begin{aligned}
& \int_{\hat{T}}^{t} e^{2 b \int_{s}^{t} z\left(\vartheta_{\tau-t} \omega\right) d \tau+\delta_{0}(s-t)}\left\|\nabla v\left(s, \vartheta_{-t} \omega, v_{0}\left(\vartheta_{-t} \omega\right)\right)\right\|^{2} d s \\
& \leq e^{2 b \int_{0}^{t} z\left(\vartheta_{s-t} \omega\right) d s-\delta_{0} t}\left\|v_{0}\left(\vartheta_{-t} \omega\right)\right\|^{2}+\frac{\|f\|^{2}+\delta_{0}\|\tilde{f}\|^{2}}{\delta_{0}} \int_{0}^{\hat{T}} e^{-2 b z\left(\vartheta_{s-t} \omega\right)+2 b \int_{s}^{t} z\left(\vartheta_{s-t} \omega\right) d s-\delta_{0}(s-t)} d s \\
& \quad+\frac{\|f\|^{2}+\delta_{0}\|\tilde{f}\|^{2}}{\delta_{0}} \int_{\hat{T}}^{t} e^{-2 b z\left(\vartheta_{s-t} \omega\right)+2 b \int_{s}^{t} z\left(\vartheta_{\tau-t} \omega\right) d \tau+\delta_{0}(s-t)} d s \\
& \leq e^{2 b \int_{0}^{t} z\left(\vartheta_{s-t} \omega\right) d s-\delta_{0} t}\left\|v_{0}\left(\vartheta_{-t} \omega\right)\right\|^{2}+\frac{\|f\|^{2}+\delta_{0}\|\tilde{f}\|^{2}}{\delta_{0}} \int_{0}^{t} e^{-2 b z\left(\vartheta_{s-t} \omega\right)+2 b \int_{s}^{t} z\left(\vartheta_{\tau-t} \omega\right) d \tau+\delta_{0}(s-t)} d s \\
& \leq e^{2 b \int_{-t}^{0} z\left(\vartheta_{s} \omega\right) d s-\delta_{0} t}\left\|v_{0}\left(\vartheta_{-t} \omega\right)\right\|^{2}+\frac{\|f\|^{2}+\delta_{0}\|\tilde{f}\|^{2}}{\delta_{0}} \int_{-t}^{0} e^{-2 b z\left(\vartheta_{s} \omega\right)+2 b \int_{s}^{0} z\left(\vartheta_{\tau} \omega\right) d \tau+\delta_{0} s} d s .
\end{aligned}
$$

Replacing $\hat{T}$ by $t$ and $t$ by $t+1$ in (50), we have

$$
\begin{aligned}
& \int_{t}^{t+1} e^{2 b \int_{s}^{t+1} z\left(\vartheta_{\tau-t-1} \omega\right) d \tau+\delta_{0}(s-t-1)}\left\|\nabla v\left(s, \vartheta_{-t-1} \omega, v_{0}\left(\vartheta_{-t-1} \omega\right)\right)\right\|^{2} d s \\
& \leq e^{2 b \int_{-t-1}^{0} z\left(\vartheta_{s} \omega\right) d s-\delta_{0}(t+1)}\left\|v_{0}\left(\vartheta_{-t-1} \omega\right)\right\|^{2}+\frac{\|f\|^{2}+\delta_{0}\|\tilde{f}\|^{2}}{\delta_{0}} \int_{-t-1}^{0} e^{-2 b z\left(\vartheta_{s} \omega\right)+2 b \int_{s}^{0} z\left(\vartheta_{\tau} \omega\right) d \tau+\delta_{0} s} d s .
\end{aligned}
$$

For $s \in[t, t+1]$, to yield that

$$
\begin{aligned}
& \int_{t}^{t+1} e^{2 b \int_{s}^{t+1} z\left(\vartheta_{\tau-t-1} \omega\right) d \tau+\delta_{0}(s-t-1)}\left\|\nabla v\left(s, \vartheta_{-t-1} \omega, v_{0}\left(\vartheta_{-t-1} \omega\right)\right)\right\|^{2} d s \\
& \geq \int_{t}^{t+1} e^{-2 b \max _{0 \leq \tau \leq 1}\left|z\left(\vartheta_{\tau} \omega\right)\right|-\delta_{0}}\left\|\nabla v\left(s, \vartheta_{-t-1} \omega, v_{0}\left(\vartheta_{-t-1} \omega\right)\right)\right\|^{2} d s .
\end{aligned}
$$

By the property of $z(\omega)$ and temperedness of $\left\|v_{0}(\omega)\right\|$, there exists $T_{B}(\omega)>0$ such that for all $t \geq T_{B}(\omega)$, from (51) and (52), we find that

$$
\begin{aligned}
& \int_{t}^{t+1}\left\|\nabla v\left(s, \vartheta_{-t-1} \omega, v_{0}\left(\vartheta_{-t-1} \omega\right)\right)\right\|^{2} d s \\
& \leq e^{2 b \int_{-t-1}^{0} z\left(\vartheta_{s} \omega\right) d s+2 b \max _{0 \leq \tau \leq 1}\left|z\left(\vartheta_{\tau} \omega\right)\right|-\delta_{0} t}\left\|v_{0}\left(\vartheta_{-t-1} \omega\right)\right\|^{2} \\
& \quad+\frac{\|f\|^{2}+\delta_{0}\|\tilde{f}\|^{2}}{\delta_{0}} \int_{-t-1}^{0} e^{-2 b z\left(\vartheta_{s} \omega\right)+2 b \max _{0 \leq \tau \leq 1}\left|z\left(\vartheta_{\tau} \omega\right)\right|+2 b \int_{s}^{0} z\left(\vartheta_{\tau} \omega\right) d \tau+\delta_{0}(s+1)} d s \\
& \leq 1+\frac{\|f\|^{2}+\delta_{0}\|\tilde{f}\|^{2}}{\delta_{0}} \int_{-\infty}^{0} e^{-2 b z\left(\vartheta_{s} \omega\right)+2 b e \max _{0 \leq \tau \leq 1}\left|z\left(\vartheta_{\tau} \omega\right)\right|+2 b \int_{s}^{0} z\left(\vartheta_{\tau} \omega\right) d \tau+\delta_{0}(s+1)} d s=\tilde{R_{1}}(\omega) .
\end{aligned}
$$

It is easy to check that $\tilde{R_{1}}(\omega)$ is tempered. This completes the proof.

Lemma 8. Assume that $f^{j}, f \in L^{2}\left(\mathbb{R}^{n}\right)$, and (3)-(5) hold. Let $\{B(\omega)\} \in \mathfrak{D}$ and $v_{0}(\omega) \in B(\omega)$. Then, for any $\zeta>0$, there exist $\tilde{T}=\tilde{T}(\zeta, \omega, B)>0$ and $\tilde{K}=\tilde{K}(\zeta, \omega)>0$, such that the solution $\phi$ of Equation (16) satisfies for P-a.e. $\omega \in \Omega, \forall t \geq \tilde{T}$,

$$
\int_{|x| \geq \tilde{R}}\left|\phi\left(t, \vartheta_{-t} \omega, v_{0}\left(\vartheta_{-t} \omega\right)\right)\right|^{2} d x \leq \zeta .
$$

Proof. We first need to define a smooth function $\sigma(\cdot)$ from $\mathbb{R}^{+}$into $[0,1]$ such that $\sigma(\cdot)=0$ on $[0,1]$ and $\sigma(\cdot)=1$ on $[2,+\infty)$, which evidently implies that there is a positive constant $c$ such that the $\left|\sigma^{\prime}(s)\right| \leq c$ for all 
$s \geq 0$. For convenience, we write $\sigma_{\kappa}=\sigma\left(\frac{|x|^{2}}{\kappa^{2}}\right)$. Now multiplying equation (16) with $\sigma_{\kappa} v$ and integrating over $\mathbb{R}^{n}$, we have

$$
\begin{aligned}
& \frac{1}{2} \frac{d}{d t} \int_{\mathbb{R}^{n}} \sigma_{\kappa}|v|^{2} d x+\lambda \int_{\mathbb{R}^{n}} \sigma_{\mathcal{K}}|v|^{2} d x \\
& =\int_{\mathbb{R}^{n}}(\Delta v) \sigma_{\kappa} v d x+b z\left(\vartheta_{t} \omega\right) \int_{\mathbb{R}^{n}} \sigma_{\mathcal{K}}|v|^{2} d x-e^{-b z\left(\vartheta_{t} \omega\right)}\left(\int_{\mathbb{R}^{n}} \sigma_{\kappa} g(u) v d x+\int_{\mathbb{R}^{n}} \sigma_{\kappa} f v d x+\int_{\mathbb{R}^{n}} D_{j} f^{j} \sigma_{\kappa} v d x\right),
\end{aligned}
$$

where

$$
\begin{aligned}
\int_{\mathbb{R}^{n}}(\Delta v) \sigma_{\kappa} v d x & =-\int_{\mathbb{R}^{n}}|\nabla v|^{2} \sigma_{\kappa} d x-\int_{\mathbb{R}^{n}} v \sigma_{\kappa}^{\prime} \frac{2 x}{\mathcal{K}^{2}}(\nabla v) d x \leq-\int_{\mathbb{R}^{n}}|\nabla v|^{2} \sigma_{\kappa} d x-\int_{\kappa \leq|x| \leq \sqrt{2} \kappa} v \sigma_{\kappa}^{\prime} \frac{2 x}{\kappa^{2}}(\nabla v) d x \\
& \leq-\int_{\mathbb{R}^{n}}|\nabla v|^{2} \sigma_{\kappa} d x+\frac{2 \sqrt{2}}{\kappa} \int_{\kappa \leq|x| \leq \sqrt{2} \kappa}|v| \cdot\left|\sigma_{\kappa}^{\prime}\right| \cdot|\nabla v| d x \\
& \leq-\int_{\mathbb{R}^{n}}|\nabla v|^{2} \sigma_{\kappa} d x+\frac{2 \sqrt{2} c}{\kappa} \int_{\mathbb{R}^{n}}|v| \cdot|\nabla v| d \leq-\int_{\mathbb{R}^{n}}|\nabla v|^{2} \sigma_{\kappa} d x+\frac{C_{0}}{\kappa}\left(\|v\|^{2}+\|\nabla v\|^{2}\right)
\end{aligned}
$$

where $C_{0}$ is a non-negative constant. Now by condition (4), we get

$$
e^{-b z\left(\vartheta_{t} \omega\right)} \int_{\mathbb{R}^{n}} \sigma_{\kappa} g(u) v d x=e^{-2 b z\left(\vartheta_{t} \omega\right)} \int_{\mathbb{R}^{n}} \sigma_{\kappa} g(u) u d x \geq \beta_{2} e^{b(p-2) z\left(\vartheta_{t} \omega\right)} \int_{\mathbb{R}^{n}} \sigma_{\kappa}|v|^{p} d x-\delta_{2} \int_{\mathbb{R}^{n}} \sigma_{\kappa}|v|^{2} d x .
$$

For the fourth term on the right-hand side of (55), we have that

$$
e^{-b z\left(\vartheta_{t} \omega\right)} \int_{\mathbb{R}^{n}} \sigma_{\kappa} f v d x \leq \frac{\delta_{0}}{2} \int_{\mathbb{R}^{n}} \sigma_{\kappa}|v|^{2} d x+\frac{1}{2 \delta_{0}} e^{-2 b z\left(\vartheta_{t} \omega\right)} \int_{\mathbb{R}^{n}} \sigma_{\kappa}|f|^{2} d x
$$

Next, we estimate the last term on the right-hand side of (55), we get that

$$
\begin{aligned}
e^{-b z\left(\vartheta_{t} \omega\right)} \int_{\mathbb{R}^{n}} D_{j} f^{j} \sigma_{\mathcal{\kappa}} v d x & =-e^{-b z\left(\vartheta_{t} \omega\right)} \int_{\mathbb{R}^{n}} \tilde{f} \frac{2 x}{\mathcal{K}^{2}} \sigma_{\kappa}^{\prime} v d x-e^{-b z\left(\vartheta_{t} \omega\right)} \int_{\mathbb{R}^{n}} \sigma_{\mathcal{\kappa}} \tilde{f}(\nabla v) d x \\
& \leq \frac{\mathcal{C}_{0}}{\mathcal{\kappa}} e^{-b z\left(\vartheta_{t} \omega\right)} \int_{\mathbb{R}^{n}}|\tilde{f}||v| d x+e^{-b z\left(\vartheta_{t} \omega\right)} \int_{\mathbb{R}^{n}}|\tilde{f}| \sigma_{\mathcal{K}}|\nabla v| d x \\
& \leq \frac{\mathcal{C}_{1}}{\kappa}\left(\|\tilde{f}\|^{2}+\|v\|^{2}\right)+\frac{\delta_{0}}{2} e^{-2 b z\left(\vartheta_{t} \omega\right)} \int_{\mathbb{R}^{n}} \sigma_{\kappa}|\tilde{f}|^{2} d x+\frac{1}{2 \delta_{0}} \int_{\mathbb{R}^{n}} \sigma_{\kappa}|\nabla v|^{2} d x
\end{aligned}
$$

where $\mathcal{C}_{1}$ is a non-negative constant. Then inserting (56) - (59) into (55) to see that

$$
\begin{aligned}
& \frac{d}{d t} \int_{\mathbb{R}^{n}} \sigma_{\kappa}|v|^{2} d x-\left(2 b z\left(\vartheta_{t} \omega\right)-\delta_{0}\right) \int_{\mathbb{R}^{n}} \sigma_{\mathcal{K}}|v|^{2} d x+\int_{\mathbb{R}^{n}}|\nabla v|^{2} \sigma_{\kappa} d x+2 \beta_{2} e^{b(p-2) z\left(\vartheta_{t} \omega\right)} \int_{\mathbb{R}^{n}} \sigma_{\mathcal{\kappa}}|v|^{p} d x \\
& \leq \frac{1}{\delta_{0}} e^{-2 b z\left(\vartheta_{t} \omega\right)} \int_{\mathbb{R}^{n}} \sigma_{\mathcal{K}}\left(|f|^{2}+\delta_{0}|\tilde{f}|^{2}\right) d x+\frac{\mathcal{C}_{2}}{\mathcal{\kappa}}\|\tilde{f}\|^{2}+\frac{\mathcal{C}_{3}}{\mathcal{K}}\|v\|^{2}+\frac{\mathcal{C}_{4}}{\mathcal{K}}\|\nabla v\|^{2}
\end{aligned}
$$

where $\mathcal{C}_{2}, \mathcal{C}_{3}$ and $\mathcal{C}_{4}$ are non-negative constants. Hence, we can rewrite (60) as

$$
\begin{aligned}
& \frac{d}{d t} \int_{\mathbb{R}^{n}} \sigma_{\mathcal{K}}|v|^{2} d x-\left(2 b z\left(\vartheta_{t} \omega\right)-\delta_{0}\right) \int_{\mathbb{R}^{n}} \sigma_{\kappa}|v|^{2} d x \\
& \leq \frac{1}{\delta_{0}} e^{-2 b z\left(\vartheta_{t} \omega\right)} \int_{\mathbb{R}^{n}} \sigma_{\mathcal{K}}\left(|f|^{2}+\delta_{0}|\tilde{f}|^{2}\right) d x+\frac{\mathcal{C}_{2}}{\kappa}\|\tilde{f}\|^{2}+\frac{\mathcal{C}_{3}}{\kappa}\|v\|^{2}+\frac{\mathcal{C}_{4}}{\kappa}\|\nabla v\|^{2} .
\end{aligned}
$$

By applying the Gronwall's lemma to (61), for every $t \geq \hat{T}$, we find that

$$
\begin{aligned}
& \int_{\mathbb{R}^{n}} \sigma_{\mathcal{K}}\left|v\left(t, \omega, v_{0}(\omega)\right)\right|^{2} d x \\
& \leq e^{2 b \int_{\hat{T}}^{t} z\left(\vartheta_{\tau} \omega\right) d \tau-\delta_{0}(t-\hat{T})} \int_{\mathbb{R}^{n}} \sigma_{\kappa}\left|v\left(\hat{T}, \omega, v_{0}(\omega)\right)\right|^{2} d x+\frac{1}{\delta_{0}} \int_{\hat{T}}^{t} e^{2 b \int_{s}^{t} z\left(\vartheta_{\tau} \omega\right) d \tau-\delta_{0}(t-s)-2 b z\left(\vartheta_{s} \omega\right)} \int_{\mathbb{R}^{n}} \sigma_{\kappa}\left(|f|^{2}+\delta_{0}|\tilde{f}|^{2}\right) d x \\
& +\frac{\mathcal{C}_{3}}{\kappa} \int_{\hat{T}}^{t} e^{2 b \int_{s}^{t} z\left(\vartheta_{\tau} \omega\right) d \tau-\delta_{0}(t-s)}\left\|v\left(s, \omega, v_{0}(\omega)\right)\right\|^{2} d s+\frac{\mathcal{C}_{4}}{\kappa} \int_{\hat{T}}^{t} e^{2 b \int_{s}^{t} z\left(\vartheta_{\tau} \omega\right) d \tau-\delta_{0}(t-s)}\left\|\nabla v\left(s, \omega, v_{0}(\omega)\right)\right\|^{2} d s \\
& +\frac{\mathcal{C}_{2}}{\kappa} \int_{\hat{T}}^{t} e^{2 b \int_{s}^{t} z\left(\vartheta_{\tau} \omega\right) d \tau-\delta_{0}(t-s)}\|\tilde{f}\|^{2} d s .
\end{aligned}
$$


Then, substituting $\omega$ by $\vartheta_{-t} \omega$ into (62), we have that

$$
\begin{aligned}
& \int_{\mathbb{R}^{n}} \sigma_{\mathcal{K}}\left|v\left(t, \vartheta_{-t} \omega, v_{0}\left(\vartheta_{-t} \omega\right)\right)\right|^{2} d x \\
& \leq e^{2 b \int_{\hat{T}}^{t} z\left(\vartheta_{\tau-t} \omega\right) d \tau-\delta_{0}(t-\hat{T})} \int_{\mathbb{R}^{n}} \sigma_{\mathcal{K}}\left|v\left(\hat{T}, \vartheta_{-t} \omega, v_{0}\left(\vartheta_{-t} \omega\right)\right)\right|^{2} d x \\
& \quad+\frac{1}{\delta_{0}} \int_{\hat{T}}^{t} e^{2 b \int_{s}^{t} z\left(\vartheta_{\tau-t} \omega\right) d \tau-\delta_{0}(t-s)-2 b z\left(\vartheta_{s-t} \omega\right)} \int_{\mathbb{R}^{n}} \sigma_{\mathcal{K}}\left(|f|^{2}+\delta_{0}|\tilde{f}|^{2}\right) d x d s \\
& \quad+\frac{\mathcal{C}_{3}}{\mathcal{\kappa}} \int_{\hat{T}}^{t} e^{2 b \int_{s}^{t} z\left(\vartheta_{\tau-t} \omega\right) d \tau-\delta_{0}(t-s)}\left\|v\left(s, \vartheta_{-t} \omega, v_{0}\left(\vartheta_{-t} \omega\right)\right)\right\|^{2} d s+ \\
& \quad+\frac{\mathcal{C}_{4}}{\mathcal{\kappa}} \int_{\hat{T}}^{t} e^{2 b \int_{s}^{t} z\left(\vartheta_{\tau-t} \omega\right) d \tau-\delta_{0}(t-s)}\left\|\nabla v\left(s, \vartheta_{-t} \omega, v_{0}\left(\vartheta_{-t} \omega\right)\right)\right\|^{2} d s+\frac{\mathcal{C}_{2}}{\kappa} \int_{\hat{T}}^{t} e^{2 b \int_{s}^{t} z\left(\vartheta_{\tau-t} \omega\right) d \tau-\delta_{0}(t-s)}\|\tilde{f}\|^{2} d s .
\end{aligned}
$$

Then, we estimate every term on the right-hand side of (63). Firstly by Equation (25), replacing $t$ by $\hat{T}$ and $\omega$ by $\vartheta_{-t} \omega$, then we get

$$
\begin{aligned}
& e^{2 b \int_{\hat{T}}^{t} z\left(\vartheta_{\tau-t} \omega\right) d \tau-\delta_{0}(t-\hat{T})} \int_{\mathbb{R}^{n}} \sigma_{\mathcal{K}}\left|v\left(\hat{T}, \vartheta_{-t} \omega, v_{0}\left(\vartheta_{-t} \omega\right)\right)\right|^{2} d x \\
& \leq e^{2 b \int_{\hat{T}}^{t} z\left(\vartheta_{\tau-t} \omega\right) d \tau-\delta_{0}(t-\hat{T})}\left(e^{2 b \int_{0}^{T} z\left(\vartheta_{s-t} \omega\right) d s-\delta_{0} \hat{T}}\left\|v_{0}\left(\vartheta_{-t} \omega\right)\right\|^{2}\right. \\
& \left.\quad+\frac{\|f\|^{2}+\delta_{0}\|\tilde{f}\|^{2}}{\delta_{0}} e^{2 b \int_{0}^{\hat{T}} z\left(\vartheta_{s-t} \omega\right) d s-\delta_{0} \hat{T}} \int_{0}^{\hat{T}} e^{-2 b z\left(\vartheta_{s-t} \omega\right)-2 b \int_{0}^{s} z\left(\vartheta_{\tau-t} \omega\right) d \tau+\delta_{0} s} d s\right) \\
& \leq e^{2 b \int_{0}^{t} z\left(\vartheta_{\tau-t} \omega\right) d \tau-\delta_{0} t}\left\|v_{0}\left(\vartheta_{-t} \omega\right)\right\|^{2}+\frac{\|f\|^{2}+\delta_{0}\|\tilde{f}\|^{2}}{\delta_{0}} e^{2 b \int_{0}^{t} z\left(\vartheta_{\tau-t} \omega\right) d \tau-\delta_{0} t} \int_{0}^{\hat{T}} e^{-2 b z\left(\vartheta_{s-t} \omega\right)-2 b \int_{0}^{s} z\left(\vartheta_{\tau-t} \omega\right) d \tau+\delta_{0} s} d s \\
& \leq e^{2 b \int_{0}^{t} z\left(\vartheta_{\tau-t} \omega\right) d \tau-\delta_{0} t}\left\|v_{0}\left(\vartheta_{-t} \omega\right)\right\|^{2}+\frac{\|f\|^{2}+\delta_{0}\|\tilde{f}\|^{2}}{\delta_{0}} \int_{0}^{T} e^{-2 b z\left(\vartheta_{s-t} \omega\right)+2 b \int_{s}^{t} z\left(\vartheta_{\tau-t} \omega\right) d \tau-\delta_{0}(t-s)} d s
\end{aligned}
$$

It easy to see that there exists $\tilde{T}_{1}=\tilde{T}_{1}(B, \zeta, \omega)>\hat{T}$, such that for all $t>\tilde{T}_{1}$, then

$$
e^{2 b \int_{\hat{T}}^{t} z\left(\vartheta_{\tau-t} \omega\right) d \tau-\delta_{0}(t-\hat{T})} \int_{\mathbb{R}^{n}} \sigma_{\mathcal{K}}\left|v\left(\hat{T}, \vartheta_{-t} \omega, v_{0}\left(\vartheta_{-t} \omega\right)\right)\right|^{2} d x \leq \zeta .
$$

For the second term on the right-hand side of (63), Since $f, \tilde{f} \in L^{2}\left(\mathbb{R}^{n}\right)$, there are $\tilde{T}_{2}=\tilde{T}_{2}(\zeta, \omega)>\hat{T}$ and $\tilde{K_{1}}=\tilde{K}_{1}(\zeta, \omega)>0$, such that for all $t>\tilde{T}_{2}$ and $\kappa>\tilde{K}_{1}$, then

$$
\begin{aligned}
& \frac{1}{\delta_{0}} \int_{\hat{T}}^{t} e^{2 b \int_{s}^{t} z\left(\vartheta_{\tau-t} \omega\right) d \tau-\delta_{0}(t-s)-2 b z\left(\vartheta_{s-t} \omega\right)} \int_{\mathbb{R}^{n}} \sigma_{\mathcal{K}}\left(|f|^{2}+\delta_{0}|\tilde{f}|^{2}\right) d x d s \\
& \leq \frac{1}{\delta_{0}} \int_{\hat{T}}^{t} e^{2 b \int_{s}^{t} z\left(\vartheta_{\tau-t} \omega\right) d \tau-\delta_{0}(t-s)-2 b z\left(\vartheta_{s-t} \omega\right)} \int_{|x| \geq \kappa}|f|^{2} d x d s+\int_{\hat{T}}^{t} e^{2 b \int_{s}^{t} z\left(\vartheta_{\tau-t} \omega\right) d \tau-\delta_{0}(t-s)-2 b z\left(\vartheta_{s-t} \omega\right)} \int_{|x| \geq \mathcal{K}}|\tilde{f}|^{2} d x d s \\
& \leq \zeta
\end{aligned}
$$

For the third term on the right-hand side of (63). By replacing $t$ by $s$ and $\omega$ by $\vartheta_{-t} \omega$ in (25), we get

$$
\begin{aligned}
& \frac{\mathcal{C}_{3}}{\mathcal{K}} \int_{\hat{T}}^{t} e^{2 b \int_{s}^{t} z\left(\vartheta_{\tau-t} \omega\right) d \tau-\delta_{0}(t-s)}\left\|v\left(s, \vartheta_{-t} \omega, v_{0}\left(\vartheta_{-t} \omega\right)\right)\right\|^{2} d s \\
& \leq \frac{\mathcal{C}_{3}}{\kappa} \int_{\hat{T}}^{t} e^{2 b \int_{s}^{t} z\left(\vartheta_{\tau-t} \omega\right) d \tau-\delta_{0}(t-s)}\left(e^{2 \int_{0}^{s} b z\left(\vartheta_{\tau-t} \omega\right) d \tau-\delta_{0} s}\left\|v_{0}\left(\vartheta_{-t} \omega\right)\right\|^{2}\right. \\
& \left.\quad+\frac{\|f\|^{2}+\delta_{0}\|\tilde{f}\|^{2}}{\delta_{0}} e^{2 b \int_{0}^{s} z\left(\vartheta_{\tau-t} \omega\right) d \tau-\delta_{0} s} \int_{0}^{s} e^{-2 b z\left(\vartheta_{\tilde{s}-t} \omega\right)-2 b \int_{0}^{\tilde{s}} z\left(\vartheta_{\tau-t} \omega\right) d \tau+\delta_{0} \tilde{s}} d \tilde{s}\right) d s \\
& \leq \frac{\mathcal{C}_{3}}{\kappa}(t-\hat{T}) e^{2 b \int_{0}^{t} z\left(\vartheta_{\tau-t} \omega\right) d \tau-\delta_{0} t}\left\|v_{0}\left(\vartheta_{-t} \omega\right)\right\|^{2}+\frac{\mathcal{C}_{3}\left(\|f\|^{2}+\delta_{0}\|\tilde{f}\|^{2}\right)}{\kappa \delta_{0}} \int_{\hat{T}}^{t} \int_{0}^{s} e^{2 b \int_{\tilde{s}}^{t} z\left(\vartheta_{\tau-t} \omega\right) d \tau-\delta_{0}(t-\tilde{s})-2 b z\left(\vartheta_{\tilde{s}-t} \omega\right)} d \tilde{s} d s
\end{aligned}
$$

Then, by $f, \tilde{f} \in L^{2}\left(\mathbb{R}^{n}\right)$, there exist $\tilde{T}_{3}=\tilde{T}_{3}(B, \zeta, \omega)>\hat{T}$ and $\tilde{K}_{2}=\tilde{K}_{2}(\zeta, \omega)>0$, such that for all $t>\tilde{T}_{3}$ and $\kappa>\tilde{K}_{2}$, we see that

$$
\frac{\mathcal{C}_{3}}{\kappa} \int_{\hat{T}}^{t} e^{2 b \int_{s}^{t} z\left(\vartheta_{\tau-t} \omega\right) d \tau-\delta_{0}(t-s)}\left\|v\left(s, \vartheta_{-t} \omega, v_{0}\left(\vartheta_{-t} \omega\right)\right)\right\|^{2} d s \leq \zeta .
$$


Next, we estimate the fourth term on the right-hand side of (63). Since $f, \tilde{f} \in L^{2}\left(\mathbb{R}^{n}\right)$, by using (50), there exist $\tilde{T}_{4}=\tilde{T}_{4}(B, \zeta, \omega)>\hat{T}$ and $\tilde{K}_{3}=\tilde{K}_{3}(\zeta . \omega)>0$, such that for all $t>\tilde{T}_{4}$ and $\kappa>\tilde{K}_{3}$, we get that

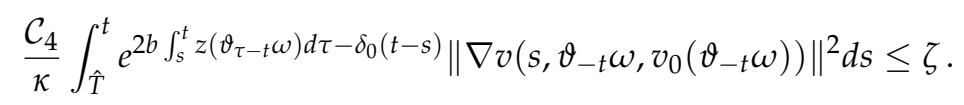

Finally, we estimate the last term on the right-hand side of (63). Since $\tilde{f} \in L^{2}\left(\mathbb{R}^{n}\right)$, there exist $\tilde{T}_{5}=$ $\tilde{T}_{5}(\zeta, \omega)>\hat{T}$ and $\tilde{K}_{4}=\tilde{K}_{4}(\zeta . \omega)>0$, such that for all $t>\tilde{T}_{5}$ and $\kappa>\tilde{K}_{4}$, we obtain that

$$
\frac{\mathcal{C}_{2}}{\mathcal{K}} \int_{\hat{T}}^{t} e^{2 b \int_{s}^{t} z\left(\vartheta_{\tau-t} \omega\right) d \tau-\delta_{0}(t-s)}\|\tilde{f}\|^{2} d s \leq \zeta
$$

By letting

$$
\begin{aligned}
\tilde{T} & =\max \left\{\tilde{T}_{1}, \tilde{T}_{2}, \tilde{T}_{3}, \tilde{T}_{4}, \tilde{T}_{5}\right\}, \\
\tilde{K} & =\max \left\{\tilde{K}_{1}, \tilde{K}_{2}, \tilde{K}_{3}, \tilde{K}_{4}\right\} .
\end{aligned}
$$

Then, inserting Equations (65)-(66),(68)-(70) into Equation (63), for all $t>\tilde{T}$ and $\kappa>\tilde{K}$, we obtain that

$$
\int_{\mathbb{R}^{n}} \sigma_{\kappa}\left|v\left(t, \vartheta_{-t} \omega, v_{0}\left(\vartheta_{-t} \omega\right)\right)\right|^{2} d x \leq 5 \zeta
$$

which shows that

$$
\int_{|x| \geq \tilde{K}}\left|\phi\left(t, \vartheta_{-t} \omega, v_{0}\left(\vartheta_{-t} \omega\right)\right)\right|^{2} d x \leq 5 \zeta
$$

The proof is concluded.

Theorem 4. Assume that $f^{j}, f \in L^{2}\left(\mathbb{R}^{n}\right)$, and (3)-(5) hold. The random dynamical system $\{\phi(t)\}_{t \geq 0}$ generated by the solution of Equation (16) with initial data $v_{0} \in L^{2}\left(\mathbb{R}^{n}\right)$. Then $\{\phi(t)\}_{t \geq 0}$ has a $\left(L^{2}\left(\mathbb{R}^{n}\right), L^{2}\left(\mathbb{R}^{n}\right)\right)$-random attractor, which is nonempty, compact, invariant in $L^{2}\left(\mathbb{R}^{n}\right)$ and attracts every bounded subset of $L^{2}\left(\mathbb{R}^{n}\right)$ with respect to $L^{2}\left(\mathbb{R}^{n}\right)$ norm.

Proof. From Lemmas 5, 6 and 8, we know that all the conditions in Corollary 1, are satisfied.

In the following, we will give the asymptotic a priori estimates of $\{\phi(t)\}_{t \geq 0}$ with respect to $L^{p}$-norm, which play a crucial role in the proof of the $\left(L^{2}\left(\mathbb{R}^{n}\right), L^{p}\left(\mathbb{R}^{n}\right)\right)$-asymptotic compactness.

Lemma 9. Assume that $f^{j}, f \in L^{2}\left(\mathbb{R}^{n}\right)$, and (3)-(5) hold. Let $\left\{B_{p}(\omega)\right\} \in \mathfrak{D}$ and $v_{0}(\omega) \in B_{p}(\omega)$. Then, for any $\zeta>0$, there exist a random constant $\tilde{T}=\tilde{T}\left(\zeta, \omega, B_{p}\right)>0$ and $\tilde{M}=\tilde{M}(\zeta, \omega)>0$, such that the solution $\phi$ of equation (16) satisfies for P-a.e. $\omega \in \Omega, \forall t \geq \tilde{T}$,

$$
\int_{\Omega(|\phi(t)| \geq \tilde{M})}\left|\phi\left(t, \vartheta_{-t} \omega, v_{0}\left(\vartheta_{-t} \omega\right)\right)\right|^{p} d x \leq \epsilon \zeta
$$

where $\left\{B_{p}(\omega)\right\}$ is the $\left(L^{2}\left(\mathbb{R}^{n}\right), L^{p}\left(\mathbb{R}^{n}\right)\right)$-bounded absorbing set obtained in the preceding lemma, and constant $\epsilon$ is the independent of $\tilde{M}, \tilde{T}$ and $\zeta$.

Proof. For any fixed $\zeta>0$, first, we know that there exists $\varrho>0$ such that if $e \subset \mathbb{R}^{n}$ and $m(e) \leq \varrho$, then

$$
\int_{e}\left|f^{j}(x)\right|^{2} d x<\zeta, \quad 1 \leq j \leq n
$$

and

$$
\int_{e}|f(x)|^{2} d x<\zeta
$$

Furthermore, by Lemmas 1, 2 and Theorem 4, there exist $\tilde{T}=\tilde{T}\left(\zeta, \omega, B_{p}\right)>0$ and $\tilde{M}_{1}=\tilde{M}(\zeta, \omega)>0$, such that for every $v_{0}(\omega) \in B(\omega)$ and $t \geq T$, we get

$$
m\left(\mathbb{R}^{n}\left(|\phi(t)| \geq \tilde{M}_{1}\right)\right) \leq\{\zeta, \varrho\}
$$


and

$$
\int_{\mathbb{R}^{n}\left(|\phi(t)| \geq \tilde{M}_{1}\right)}\left|\phi\left(t, \vartheta_{-t} \omega, v_{0}\left(\vartheta_{-t} \omega\right)\right)\right|^{2} d x \leq 8 \zeta
$$

Additionally, it follows by (4) that $f(s) \geq 0$ provided that $s>\left(\delta_{2} / \beta_{2}\right)^{1 /(p-2)}$. Let $\tilde{M}=$ $\max \left\{\tilde{M}_{1},\left(\delta_{2} / \beta_{2}\right)^{1 /(p-2)}\right\}$ and $t \geq T$. Multiplying equation (16) with $(v-\tilde{M})_{+}$and integrating over $\mathbb{R}^{n}$, we find that

$$
\begin{aligned}
& \left.\frac{1}{2} \frac{d}{d t} \int_{\mathbb{R}^{n}}\left|(v-\tilde{M})_{+}\right|^{2} d x+\int_{\mathbb{R}^{n}(v \geq \tilde{M})}\left|\nabla(v-\tilde{M})_{+}\right|^{2} d x+\left(\lambda-b z\left(\vartheta_{t} \omega\right)\right) \int_{\mathbb{R}^{n}} v \cdot(v-\tilde{M})_{+}\right) d x \\
& +e^{-b z\left(\vartheta_{t} \omega\right)} \int_{\mathbb{R}^{n}} g(u)(v-\tilde{M})_{+} d x=-e^{-b z\left(\vartheta_{t} \omega\right)} \int_{\mathbb{R}^{n}} \tilde{f} \cdot \nabla(v-\tilde{M})_{+} d x+e^{-b z\left(\vartheta_{t} \omega\right)}\left(f,(v-\tilde{M})_{+}\right)
\end{aligned}
$$

where $\left.(v-\tilde{M})_{+}\right)$denotes the non-negative part of $(v-\tilde{M})$, that is

$$
(v-\tilde{M})_{+}= \begin{cases}v-\tilde{M} & v \geq \tilde{M}_{+} \\ 0 & v \leq \tilde{M}_{+} .\end{cases}
$$

Let $\mathbb{R}_{1}^{n}=\mathbb{R}^{n}(v \geq \tilde{M})$, then, we can rewrite (79) as

$$
\begin{aligned}
& \left.\frac{1}{2} \frac{d}{d t} \int_{\mathbb{R}^{n}}\left|(v-\tilde{M})_{+}\right|^{2} d x+\int_{\mathbb{R}_{1}^{n}}|\nabla v|^{2} d x+\left(\lambda-b z\left(\vartheta_{t} \omega\right)\right) \int_{\mathbb{R}^{n}} v \cdot(v-\tilde{M})_{+}\right) d x+e^{-b z\left(\vartheta_{t} \omega\right)} \int_{\mathbb{R}_{1}^{n}} g(u)(v-\tilde{M}) d x \\
& =-e^{-b z\left(\vartheta_{t} \omega\right)} \int_{\mathbb{R}_{1}^{n}} \tilde{f} \cdot \nabla v d x+e^{-b z\left(\vartheta_{t} \omega\right)} \int_{\mathbb{R}_{1}^{n}} f(v-\tilde{M}) d x
\end{aligned}
$$

By the Cauchy inequality and the Hölder inequality, it yields

$$
\begin{aligned}
& \frac{d}{d t}\left\|(v-\tilde{M})_{+}\right\|^{2}+\epsilon\left(\int_{\mathbb{R}_{1}^{n}}|\nabla v|^{2} d x+\int_{\mathbb{R}_{1}^{n}} v \cdot(v-\tilde{M})_{+} d x+\int_{\mathbb{R}_{1}^{n}} g(u)(u-\tilde{M}) d x\right) \\
& \leq \epsilon\left(\int_{\mathbb{R}_{1}^{n}}|\tilde{f}|^{2} d x+\int_{\mathbb{R}_{1}^{n}}|f|^{2} d x\right) .
\end{aligned}
$$

Then inserting (75)-(78) into (81) and integrating from $t$ to $t+1$, we find that

$$
\int_{t}^{t+1}\left(\int_{\mathbb{R}_{1}^{n}}|\nabla v|^{2} d x+\int_{\mathbb{R}_{1}^{n}}|v|^{2} d x+\int_{\mathbb{R}_{1}^{n}} g(u)(u-\tilde{M}) d x\right) d x \leq \epsilon \zeta .
$$

Hence,

$$
\int_{t}^{t+1}\left(\int_{\mathbb{R}^{n}(v \geq 2 \tilde{M})}|\nabla v+\tilde{f}|^{2} d x+\int_{\mathbb{R}^{n}(v \geq 2 \tilde{M})}|v|^{2} d x+\int_{\mathbb{R}^{n}(v \geq 2 \tilde{M})} g(u) u d x\right) d x \leq \epsilon \zeta .
$$

On the other hand, multiplying Equation (16) with $\left[(v-2 \tilde{M})_{+}\right]_{t}$ and denote $\mathbb{R}^{n}(v \geq 2 \tilde{M})$ by $\mathbb{R}_{2}^{n}$, then we have

$$
\frac{d}{d t}\left(\int_{\mathbb{R}_{2}^{n}}|\nabla v+\tilde{f}|^{2} d x+\int_{\mathbb{R}_{2}^{n}}|v|^{2} d x+\int_{\mathbb{R}_{2}^{n}} g(u) d x\right) d x \leq \epsilon \zeta
$$

in the same method as in proving (41).

Combining with (83)- (84), by using the uniform Gronwall lemma, we see that

$$
\int_{\mathbb{R}_{2}^{n}}|\nabla v+\tilde{f}|^{2} d x+\int_{\mathbb{R}_{2}^{n}}|v|^{2} d x+\int_{\mathbb{R}_{2}^{n}} g(u) d x \leq \epsilon \zeta .
$$

Hence, we can rewrite (85) as

$$
\int_{\mathbb{R}_{2}^{n}}|\nabla v|^{2} d x \leq 2 \int_{\mathbb{R}_{2}^{n}}|\nabla v+\tilde{f}|^{2} d x+2 \int_{\mathbb{R}_{2}^{n}}|\tilde{f}|^{2} d x \leq \epsilon \zeta
$$

and

$$
\int_{\mathbb{R}_{2}^{n}} g(u) d x=\int_{\mathbb{R}_{2}^{n}} g\left(e^{b z\left(\vartheta_{t} \omega\right)} v\right) d x
$$


Since $g$ is Lipschitz

$$
\int_{\mathbb{R}_{2}^{n}} g\left(e^{b z\left(\vartheta_{t} \omega\right)} v\right) d x=\int_{\mathbb{R}_{2}^{n}}\left[g\left(e^{b z\left(\vartheta_{t} \omega\right)} v\right)-g(0)\right] d x \leq C \int_{\mathbb{R}_{2}^{n}} e^{b z\left(\vartheta_{t} \omega\right)} \cdot|v| d x \leq C \epsilon \zeta .
$$

Repeating the same steps as above and taking $(v+\tilde{M})_{-}$and $\left[(v+2 \tilde{M})_{-}\right]_{t}$ instead of $(v-\tilde{M})_{+}$and $[(v-$ $\left.2 \tilde{M})_{+}\right]_{t}$, respectively, we conclude that

$$
\int_{\mathbb{R}^{n}(v \leq-2 \tilde{M})}|\nabla v|^{2} d x \leq \epsilon \zeta
$$

and

$$
\int_{\mathbb{R}^{n}(v \leq-2 \tilde{M})} g(u) d x \leq \epsilon \zeta
$$

Then from (86) - (89), we conclude that

$$
\int_{\mathbb{R}^{n}(|v| \geq 2 \tilde{M})} \mid \nabla v\left(\left.(t)\right|^{2} d x \leq \epsilon \zeta\right.
$$

and

$$
\int_{\mathbb{R}^{n}(|v| \geq 2 \tilde{M})} g(u(t)) d x \leq \epsilon \zeta .
$$

Thus, due to (39) and (78), Lemma 9 follows from (91).

By theorem 3, we directly get.

Theorem 5. Assume that $f^{j}, f \in L^{2}\left(\mathbb{R}^{n}\right)$, and (3)-(5) hold. The random dynamical system $\{\phi(t)\}_{t \geq 0}$ generated by the solution of Equation (16) with initial data $v_{0} \in L^{2}\left(\mathbb{R}^{n}\right)$. Then $\{\phi(t)\}_{t \geq 0}$ has a $\left(L^{2}\left(\mathbb{R}^{n}\right), L^{p}\left(\mathbb{R}^{n}\right)\right)$-random attractor, which is nonempty, compact, invariant in $L^{p}\left(\mathbb{R}^{n}\right)$ and attracts every bounded subset of $L^{2}\left(\mathbb{R}^{n}\right)$ with respect to $L^{p}\left(\mathbb{R}^{n}\right)$ norm.

Remark 2. From Theorem 2, we have that the $\left(L^{2}\left(\mathbb{R}^{n}\right), L^{2}\left(\mathbb{R}^{n}\right)\right)$-random attractor coincides with the $\left(L^{2}\left(\mathbb{R}^{n}\right), L^{p}\left(\mathbb{R}^{n}\right)\right)$-random attractor.

Author Contributions: All authors contributed equally to the writing of this paper. All authors read and approved the final manuscript.

Conflicts of Interest: "The authors declare that they have no competing interests."

\section{References}

[1] Crauel, H., \& Flandoli, F. (1994). Attractors for random dynamical systems. Probability Theory and Related Fields, 100(3), 365-393.

[2] Crauel, H., Debussche, A., \& Flandoli, F. (1997). Random attractors. Journal of Dynamics and Differential Equations, 9(2), 307-341.

[3] Arnold, L. (1998). Trends and open problems in the theory of random dynamical systems. In Probability towards 2000 (pp. 34-46). Springer, New York, NY.

[4] Caraballo, T., Kloeden, P. E., \& SchmalfuSS, B. (2004). Exponentially stable stationary solutions for stochastic evolution equations and their perturbation. Applied Mathematics and Optimization, 50(3), 183-207.

[5] Caraballo Garrido, T., Langa Rosado, J. A., \& Robinson, J. C. (2001). A stochastic pitchfork bifurcation in a reaction-diffusion equation. Proceedings-Royal Society. Mathematical, Physical and Engineering Sciences, 457 (2013), 2041-2061.

[6] Crauel, H. (2002). Random probability measures on Polish spaces (Vol. 11). CRC press.

[7] Zhou, S. (2017). Random exponential attractor for stochastic reactionÜdiffusion equation with multiplicative noise in R3. Journal of Differential Equations, 263(10), 6347-6383.

[8] Flandoli, F., \& Schmalfuss, B. (1996). Random attractors for the 3D stochastic Navier-Stokes equation with multiplicative white noise. Stochastics: An International Journal of Probability and Stochastic Processes, 59(1-2), 21-45.

[9] Zhao, C., \& Zhou, S. (2009). Sufficient conditions for the existence of global random attractors for stochastic lattice dynamical systems and applications. Journal of Mathematical Analysis and Applications, 354(1), 78-95.

[10] Mosa, F. M., Ma, Q., \& Bakhet, M. Y. (2018). Existence of random attractors for stochastic non-autonomous reaction-diffusion equation with multiplicative noise on $\mathbb{R}^{n}$. The Korean Journal of Mathematics, 26(4), 583-599. 
[11] Bates, P. W., Lu, K., \& Wang, B. (2009). Random attractors for stochastic reactionŰdiffusion equations on unbounded domains. Journal of Differential Equations, 246(2), 845-869.

[12] Wang, Z., \& Zhou, S. (2011). Random attractor for stochastic reaction-diffusion equation with multiplicative noise on unbounded domains. Journal of Mathematical Analysis and Applications, 384(1), 160-172.

[13] Sun, C. Y., \& Zhong, C. K. (2005). Attractors for the semilinear reactionÚdiffusion equation with distribution derivatives in unbounded domains. Nonlinear Analysis: Theory, Methods E Applications, 63(1), 49-65.

[14] Sun, C., Yuan, L., \& Shi, J. (2013). Higher-order integrability for a semilinear reactionÜdiffusion equation with distribution derivatives in RN. Applied Mathematics Letters, 26(9), 949-956.

[15] Ahmed, E. M., Abdelmajid, A. D., Xu, L., \& Ma, Q. (2015). Random attractors for stochastic reaction-diffusion equations with distribution derivatives on unbounded domains. Applied Mathematics, 6(10), 1790.

[16] Mosa, F. M., Ma, Q., \& Yao, X. (2019). Dynamics for the Stochastic Reaction-diffusion Equation with Distribution Derivatives and Multiplicative Noise on Rn. Asian Research Journal of Mathematics, 1-15.

[17] Adams, R. A. Sobolev Spaces, Academic Press, New York, 1975. MR0450957 (56: 9247).

[18] Ball, J. M. (2000). Continuity properties and global attractors of generalized semiflows and the Navier-Stokes equations. In Mechanics: from theory to computation (pp. 447-474). Springer, New York, NY.

[19] Ghidaglia, J. M. (1994). A Note on the Strong Convergence tovvards Attractors of Damped Forced KdV Equations. Journal of Differential Equations, 110, 356-359.

[20] Goubet, O., \& Rosa, R. M. (2002). Asymptotic smoothing and the global attractor of a weakly damped KdV equation on the real line. Journal of Differential Equations, 185(1), 25-53.

[21] Ju, N. (2000). The H1-compact global attractor for the solutions to the Navier-Stokes equations in two-dimensional unbounded domains. Nonlinearity, 13(4), 1227.

[22] Moise, I., \& Rosa, R. (1997). On the regularity of the global attractor of a weakly damped, forced Korteweg-de Vries equation. Advances in Differential Equations, 2(2), 257-296.

[23] Wang, B. (2009). Random attractors for the stochastic FitzHughÜNagumo system on unbounded domains. Nonlinear Analysis: Theory, Methods \& Applications, 71(7-8), 2811-2828.

[24] Wang, B. (2008). Random attractors for the stochastic Benjamin-Bona-Mahony equation on unbounded domains. arXiv preprint arXiv:0805.1781.

[25] Chueshov, I. (2004). Monotone random systems theory and applications. Springer.

[26] Li, J., Li, Y., \& Wang, B. (2010). Random attractors of reaction-diffusion equations with multiplicative noise in Lp. Applied mathematics and computation, 215(9), 3399-3407.

[27] Li, Y., \& Guo, B. (2008). Random attractors for quasi-continuous random dynamical systems and applications to stochastic reactionÜdiffusion equations. Journal of Differential Equations, 245(7), 1775-1800.

[28] Zhong, C., K., Yang, M., H., \& Sun, C. Y. (2006). The existence of global attractor for the norm-to-weak continuous semigroup and applications to the nonlinear reaction-diffusion equations. Journal of Differential Equations, 223, 367-399.

[29] Babin, A. V., \& Vishik, M. I. (1992). Attractors of evolution equations. Elsevier.

[30] Zhong, C., Sun, C., \& Niu, M. (2005). On the existence of global attractor for a class of infinite dimensional dissipative nonlinear dynamical systems. Chinese Annals of Mathematics, 26(03), 393-400.

[31] Fan, X. (2006). Attractors for a damped stochastic wave equation of SineÜGordon type with sublinear multiplicative noise. Stochastic Analysis and Applications, 24(4), 767-793.

[32] Duan, J., Lu, K., \& Schmalfuss, B. (2003). Invariant manifolds for stochastic partial differential equations. The Annals of Probability, 31(4), 2109-2135.

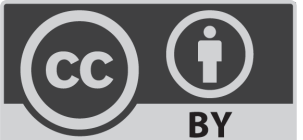

(C) 2020 by the authors; licensee PSRP, Lahore, Pakistan. This article is an open access article distributed under the terms and conditions of the Creative Commons Attribution (CC-BY) license (http://creativecommons.org/licenses/by/4.0/). 